\title{
Design and Multiplier-Less Implementation of a Class of Two-Channel PR FIR Filterbanks and Wavelets with Low System Delay
}

\author{
J. S. Mao, S. C. Chan, W. Liu, and K. L. Ho, Member, IEEE
}

\begin{abstract}
In this paper, a new method for designing two-channel PR FIR filterbanks with low system delay is proposed. It is based on the generalization of the structure previously proposed by Phoong et al. Such structurally PR filterbanks are parameterized by two functions $(\beta(z)$ and $\alpha(z))$ that can be chosen as linear-phase FIR or allpass functions to construct FIR/IIR filterbanks with good frequency characteristics. The case of using identical $\beta(z)$ and $\alpha(z)$ was considered by Phoong et al. with the delay parameter $M$ chosen as $2 N-1$. In this paper, the more general case of using different nonlinear-phase FIR functions for $\beta(z)$ and $\alpha(z)$ is studied. As the linear-phase constraint is relaxed, the lengths of $\beta(z)$ and $\alpha(z)$ are no longer restricted by the delay parameters of the filterbanks. Hence, higher stopband attenuation can still be achieved at low system delay. The design of the proposed low-delay filterbanks is formulated as a complex polynomial approximation problem, which can be solved by the Remez exchange algorithm or analytic formula with very low complexity. In addition, the orders and delay parameters can be estimated from the given filter specifications using a simple empirical formula. Therefore, low-delay two-channel PR filterbanks with flexible stopband attenuation and cutoff frequencies can be designed using existing filter design algorithms. The generalization of the present approach to the design of a class of wavelet bases associated with these low-delay filterbanks and its multiplier-less implementation using the sum of powers-of-two coefficients are also studied.
\end{abstract}

Index Terms-Filterbanks, genetic algorithm, perfect reconstruction.

\section{INTRODUCTION}

$\mathbf{P}$ ERFECT reconstruction (PR) multirate filterbanks have important applications in signal analysis, coding, and the design of wavelet bases. Fig. 1 shows the block diagram of a two-channel maximally decimated filterbank. The system is a PR system if the output $\hat{x}(n)$ is identical to the input $x(n)$ except for some constant scaling and time delay. The theory of PR filterbanks has been extensively studied in the literature [1]-[5]. A number of techniques for designing two-channel PR filterbanks are now available [1]-[10]. Because of the important application of filterbanks and wavelets in image and video coding,

Manuscript received November 9, 1999; revised August 25, 2000. This work was supported by the Research Grant Council in Hong Kong SAR. The associate editor coordinating the review of this paper and approving it for publication was Dr. Xiang-Gen Xia.

J. S. Mao is with University of Victoria, Victoria, BC, Canada

S. C. Chan, W. Liu, and K. L. Ho are with the Department of Electrical and Electronic Engineering, The University of Hong Kong, Hong Kong (e-mail klho@eee.hku.hk).

Publisher Item Identifier S 1053-587X(00)10145-X much attention has been given to the design of PR filterbanks with linear-phase response [6]-[9], [16], [30]. Nayebi et al. [10] were the first to consider the design of low-delay perfect reconstruction filterbanks. The problem was formulated as an optimization problem and was solved using the conjugate gradient algorithm. One problem with the optimization approach is that the filterbanks so obtained are in general not PR (pseudo PR). This is also a major problem of other related works based on optimization techniques [11], [13], [15], [18]-[20]. One solution to this problem is to employ filterbanks that are inherently or structurally PR. The design of such structurally PR low-delay filterbanks were recently reported in [12], [14], and [17]. Unfortunately, because of the cascading structure of the filterbank, the objective function of the unconstrained optimization is usually highly nonlinear, and the optimization program can easily get trapped in local minimum with unsatisfactory frequency characteristics.

In this paper, a new method for designing two-channel PR FIR filterbanks with low system delay is proposed. It is based on the generalization of the structure previously proposed by Phoong et al. [9]. Such structurally PR filterbanks are parameterized by two functions $(\beta(z)$ and $\alpha(z))$ that can be chosen as linear-phase FIR or allpass functions to construct FIR/IIR filterbanks with good frequency characteristics. The case of using identical $\beta(z)$ and $\alpha(z)$ was considered in [9] with the delay parameter $M$ chosen as $2 N-1$. In this paper, the more general case of using different nonlinear-phase FIR functions for $\beta(z)$ and $\alpha(z)$ is studied. As the linear-phase constraint is relaxed, the lengths of $\beta(z)$ and $\alpha(z)$ are no longer restricted by the delay parameters of the filterbanks. Hence, higher stopband attenuation can still be achieved at low system delay. The design of the proposed low-delay filterbanks is formulated as a complex polynomial approximation problem, which can be solved by the Remez exchange algorithm or analytic formula with very low complexity. As a result, low-delay two-channel PR filterbanks with flexible stopband attenuation and cutoff frequencies can be designed using existing filter design algorithms. The proposed technique is also applicable to the case where $\beta(z)$ and $\alpha(z)$ are allpass and type-II linear-phase functions, respectively. This class of passband linear-phase IIR filterbank is able to suppress the bumping problem previously found in [9] when both $\beta(z)$ and $\alpha(z)$ are chosen as allpass functions. Due to the use of the allpass and linear-phase FIR functions, the design and implementation complexities of this filterbank are also very low.

Using the proposed approach and a new method for structurally imposing the regularity condition, a new class of wavelet 
bases associated with this low-delay filterbank is obtained. Furthermore, by using the sum of powers-of-two coefficient representation and the genetic algorithm (GA), multiplier-less implementation of the proposed low-delay filterbanks are obtained. Design examples show that the GA is a very effective method for performing such discrete optimization. The multiplier-less low-delay filterbanks so obtained have good frequency characteristics and low implementation complexity. Although the primary objective of this paper is to design filterbanks with high stopband attenuation and low system delay using the Remez exchange algorithm and least squares criteria, it should be noted that the structure of Phoong et al. [9] and that in [14] can also be designed to meet other performance measure such as the coding gain. Under these circumstances, more general nonlinear optimization techniques are usually required.

The paper is organized as follows: In Section II, a brief summary of the two-channel structurally PR filterbank proposed in [9] is given together with the basic idea behind the proposed low-delay FIR filterbanks. Details of the proposed design method and several design examples are given in Section III. The design of dyadic wavelet bases derived from these two-channel FIR filterbanks is studied in Section IV, where a new method for structurally imposing the regularity condition is proposed. Section V is devoted to the multiplier-less implementation of the proposed low-delay FIR filterbanks using sum of powers-of-two coefficients (SOPOT) and the GA. Finally, conclusions are drawn in Section VI.

\section{TwO-CHANNEL STRUCTURALLY PR FILTERBANKS}

Fig. 1 shows the structure of a two-channel maximally decimated multirate filterbank. It can be shown that [1], [2] the reconstructed signal $\hat{X}(z)$ is given by

$$
\hat{X}(z)=T(z) X(z)+A(z) X(-z)
$$

where

$$
T(z)=\frac{1}{2}\left[H_{0}(z) G_{0}(z)+H_{1}(z) G_{1}(z)\right]
$$

and

$$
A(z)=\frac{1}{2}\left[H_{0}(-z) G_{0}(z)+H_{1}(-z) G_{1}(z)\right]
$$

The aliasing term $A(z)$ can be canceled if the analysis and synthesis filters are chosen as

$$
G_{0}(z)=-H_{1}(-z), \quad G_{1}(z)=H_{0}(-z)
$$

Furthermore, the filterbank will be PR if the transfer function $T(z)$ is equal to a constant multiple of signal delay

$$
T(z)=c z^{-n_{0}}
$$

Combining (2.3) and (2.4), one gets the following PR condition in $H_{0}(z)$ and $H_{1}(z)$ :

$$
T(z)=\frac{1}{2}\left[H_{0}(-z) H_{1}(z)-H_{0}(z) H_{1}(-z)\right]=c z^{-n_{0}}
$$

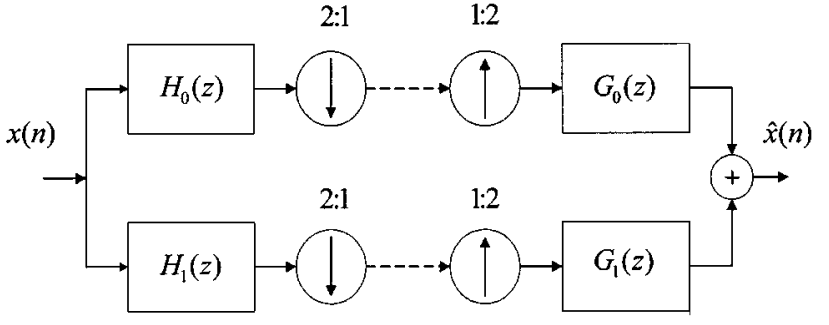

Fig. 1. Two-channel maximally decimated multirate filterbank.

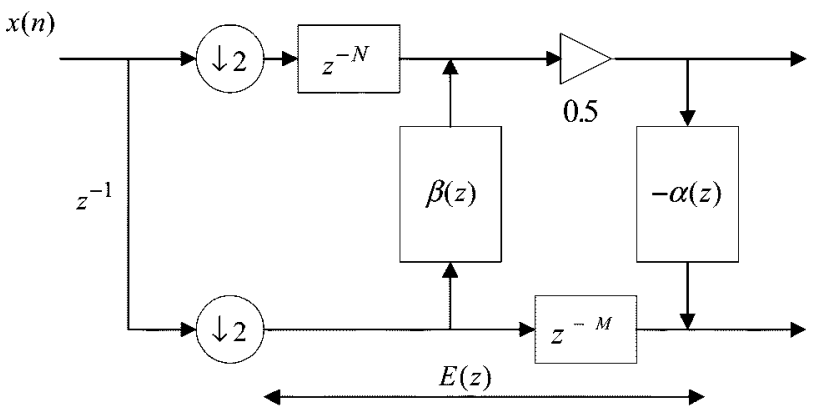

(a)

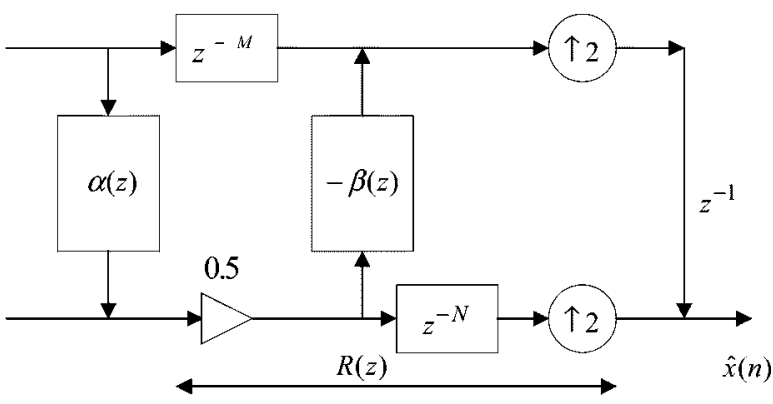

(b)

Fig. 2. Structurally PR two-channel filterbank proposed in [9].

where $n_{0}$ is an integer, and $c$ is a nonzero constant. The structurally PR two-channel FIR/IIR filterbanks proposed in [9] are shown in Fig. 2. The expressions for the analysis filters are given by

$$
H_{0}(z)=\frac{\left(z^{-2 N}+z^{-1} \beta\left(z^{2}\right)\right)}{2}
$$

and

$$
H_{1}(z)=-\alpha\left(z^{2}\right) H_{0}(z)+z^{-2 M-1} .
$$

It can be seen from (2.7) and (2.8) that (2.6) is satisfied for any choices of $\beta(z)$ and $\alpha(z)$. Therefore, FIR and IIR filterbanks can readily be realized by choosing $\beta(z)$ and $\alpha(z)$ as polynomials or rational functions. In [9], the case of using identical $\beta(z)$ and $\alpha(z)$ is studied with the delay parameter $M$ chosen as $2 N-1$. A new class of FIR and IIR filterbanks was obtained by choosing $\beta(z)$ and $\alpha(z)$ as Type-II linear-phase functions and allpass functions, respectively. The design of $\beta(z)$ [and $\alpha(z)$ ] 
can be accomplished by noting that $H_{0}(z)$ [and $H_{1}(z)$ ] will become an ideal lowpass filter if $\beta(z)$ [and $\alpha(z)$ ] has the following magnitude and phase responses

$$
\beta\left(e^{j 2 \omega}\right)= \begin{cases}e^{-j(2 N-1) \omega}, & \text { for } \omega \in[0, \pi / 2] \\ e^{-j((2 N-1) \omega \pm \pi)}, & \text { for } \omega \in(\pi / 2, \pi] .\end{cases}
$$

With this choice of $\beta\left(e^{j 2 \omega}\right), H_{0}\left(e^{j \omega}\right)$ is approximately equal to $e^{-j 2 N \omega}$ in the passband $(\omega \in[0, \pi / 2])$ and 0 in the stopband $(\omega \in[\pi / 2, \pi])$. To design a linear-phase FIR filterbank, $\beta(z)$ has to be a type-II linear-phase function with length $N_{\beta}=2 N$, which is an even number. Furthermore, if $\alpha(z)=\beta(z)$, then from (2.8), $\beta\left(e^{j 2 \omega}\right) H_{0}\left(e^{j \omega}\right)$ should approximate $e^{-j(2 M+1) \omega}$ in the stopband of $H_{1}\left(e^{j \omega}\right)$. Because $\beta\left(e^{j \omega}\right) H_{0}\left(e^{j \omega}\right)$ is very small in the passband of $H_{1}\left(e^{j \omega}\right), H_{1}\left(e^{j \omega}\right)$ will approximate a delay $e^{-j(2 M+1) \omega}$ in its passband. Since the delays of $H_{0}\left(e^{j \omega}\right)$ and $\beta\left(e^{2 j \omega}\right)$ in the interval $\omega=[0, \pi / 2]$ are $2 N$ and $2 N-1$, respectively, the delay parameter $M$ must be equal to $(2 N-1)$. It is worth noting that linear-phase FIR filterbank with $\alpha(z) \neq \beta(z)$ can also be designed by properly selecting the delay parameter $M$ and the length of $\alpha(z), N_{\alpha}$. This has been reported and studied previously in [6] and [7]. The relation between system delay $n_{0}$, the delay parameters $N$ and $M$, and the lengths of the linear-phase FIR functions $\alpha(z)$ and $\beta(z)$, $N_{\alpha}$, and $N_{\beta}$, are summarized as follows:

$N_{\beta}=2 N, \quad\left(N_{\alpha}-1\right)+2 N=2 M+1, \quad n_{0}=2 N+2 M+1$.

In summary, the delay parameters of these types of structurally PR filterbanks determine the system delay and the length of the analysis filters. It can also be observed from (2.8) and (2.10) that the delay of the highpass filter $H_{1}(z)$ is always larger than that of the lowpass filter $H_{0}(z)$. This is due to the special filterbank structure in Fig. 2. It is also interesting to note that by inverting the signs of $\beta(z)$ and $\alpha(z)$, the role of the lowpass and highpass filters in this structure can be interchanged. In fact, $H_{0}(z)$ becomes a highpass filter and $H_{1}(z)$ becomes a lowpass filter. This yields a filterbank with a highpass filter $H_{0}(z)$ having a shorter filter length and delay response than the lowpass filter $H_{1}(z)$. For simplicity, it is assumed throughout this paper that $H_{0}(z)$ is a lowpass filter and that $H_{1}(z)$ is a highpass filter. For low-delay applications [32], the length of the linear-phase FIR function might be too small to provide sufficient stopband attenuation. One solution is to relax the linear-phase requirement of $\beta(z)$ and $\alpha(z)$ and use nonlinear-phase FIR functions. The system delay in this case is still given by $n_{0}=2 N+2 M+1$. $N_{\alpha}$ and $N_{\beta}$, however, can be greater than $2(M-N+1)$ and $2 N$, unlike their linear-phase counterparts. It should be noted that the conjugate quadrature filters (CQF's) in [1], which are obtained by spectral factorization of linear-phase half band filters, are nonlinear phase. One of them is minimum-phase, and the other is maximum-phase. Other choices are possible, but the system delay is still limited by the length of the filters. Therefore, the structure considered here is more suitable for low-delay applications.

In the following section, we will consider the case where $\beta(z)$ and $\alpha(z)$ are chosen as nonlinear-phase FIR functions, and a new class of PR filterbanks with low system delay will be in- troduced. The design of such nonlinear-phase FIR functions is formulated as a complex polynomial approximation problem, which is solved using the Remez exchange algorithm and analytic formula.

\section{PROPOSED METHOD}

\section{A. Design of Lowpass Filter}

Let $\omega_{p_{0}}$ and $\omega_{s_{0}}$ be, respectively, the passband and stopband cutoff frequencies of $H_{0}(z)$. Similarly, let $\omega_{p_{1}}$ and $\omega_{s_{1}}$ be the passband and stopband cutoff frequencies of $H_{1}(z)$. Supposing that $\omega_{p_{0}}=\omega_{s_{1}}=\omega_{p}$ and $\omega_{s_{0}}=\omega_{p_{1}}=\omega_{s}$, the desired frequency response of $H_{0}(z)$ is

$$
H_{d}^{(0)}\left(e^{j \omega}\right)= \begin{cases}e^{-j 2 N \omega}, & 0 \leq \omega \leq \omega_{p} \\ 0, & \omega_{s} \leq \omega \leq \pi\end{cases}
$$

where $N$ is a positive integer. The error function in approximating $H_{d}^{(0)}(z)$ is therefore given by

$$
\begin{gathered}
E_{0}(\omega)=\frac{1}{2}\left(e^{-j 2 N \omega}+\beta\left(e^{2 j \omega}\right) e^{-j \omega}\right)-H_{d}^{(0)}\left(e^{j \omega}\right) \\
0 \leq \omega \leq \pi
\end{gathered}
$$

Using (3.1), (3.2) can be written more clearly as

$$
E_{0}(\omega)= \begin{cases}\frac{1}{2}\left(\beta\left(e^{2 j \omega}\right) e^{-j \omega}-e^{-j 2 N \omega}\right), & 0 \leq \omega \leq \omega_{p} \\ \frac{1}{2}\left(e^{-j 2 N \omega}+\beta\left(e^{2 j \omega}\right) e^{-j \omega}\right), & \omega_{s} \leq \omega \leq \pi\end{cases}
$$

Since $H_{0}(z)$ is a halfband lowpass filter, its cut-off frequen$\operatorname{cies} \omega_{p}$ and $\omega_{s}$ are placed symmetrically with respect to $\pi / 2$ [2], that is, $\omega_{p}+\omega_{s}=\pi$. Thus, it can be easily derived from (3.3) that

$$
E_{0}(\omega)=-E_{0}^{*}(\pi-\omega), \quad 0 \leq \omega \leq \omega_{p} .
$$

Although the minimization in (3.2) involves $\beta\left(e^{2 j \omega}\right)$ and is different from the conventional Chebyshev approximation, the property of the low delay halfband filter obtained in (3.4) suggests that we can minimize $E_{0}(\omega)$ either in the passband or stopband of $H_{0}(z)$, i.e., $0 \leq \omega \leq \omega_{p}$ or $\omega_{s} \leq \omega \leq \pi$. The ideal response of $\beta(z)$ can be chosen to be the same as (2.9).

For the time being, we will assume that the length of the FIR function $\beta(z), N_{\beta}$ is even. The odd value case can be obtained similarly and is summarized in Appendix A. As mentioned earlier, $\beta(z)$ will be a linear-phase function with $N_{\beta}=2 N$ in order to realize filterbanks with linear phase. In a low-delay filterbank, the required delay $N$ will, in general, be smaller than the length of $\beta(z)$, i.e., $N<N_{\beta} / 2$. First of all, let us decompose the impulse response $h_{\beta}(n)$ of $\beta(z)$ into its even and odd parts

$$
h_{\beta}(n)=h_{\beta, e}(n)+h_{\beta, o}(n)
$$

where

and

$$
h_{\beta, e}(n)=\frac{h_{\beta}(n)+h_{\beta}\left(N_{\beta}-n-1\right)}{2}
$$

$$
h_{\beta, o}(n)=\frac{h_{\beta}(n)-h_{\beta}\left(N_{\beta}-n-1\right)}{2} .
$$

As the length of $\beta(z)$ is even, $\beta_{e}(z)$ and $\beta_{o}(z)$, which are the $z$-transforms of $h_{\beta, e}(n)$ and $h_{\beta, o}(n)$, are type-II and type-IV 
linear-phase FIR functions, respectively. Therefore, they can be expressed in the following forms:

$$
\begin{aligned}
& \beta_{e}\left(e^{j \omega}\right)=e^{-j \omega\left(N_{\beta}-1\right) / 2} \cos (\omega / 2) P_{e}^{(\beta)}(\cos (\omega)) \\
& \beta_{o}\left(e^{j \omega}\right)=j e^{-j \omega\left(N_{\beta}-1\right) / 2} \sin (\omega / 2) P_{o}^{(\beta)}(\cos (\omega))
\end{aligned}
$$

where

$$
\begin{aligned}
& P_{e}^{(\beta)}(\cos (\omega))=\sum_{k=0}^{L_{\beta}} a_{k}(\cos (\omega))^{k} \\
& P_{o}^{(\beta)}(\cos (\omega))=\sum_{k=0}^{L_{\beta}} b_{k}(\cos (\omega))^{k} .
\end{aligned}
$$

Both polynomials $P_{e}^{(\beta)}(x)$ and $P_{o}^{(\beta)}(x)$ have orders $L_{\beta}$ and are equal to $\left(N_{\beta} / 2\right)-1$. Substituting (3.7) and (3.5) into (3.2), one gets

$$
\begin{aligned}
E_{0}(\omega)=\frac{1}{2} e^{-j \omega N_{\beta}}\left\{\cos (\omega) \cdot P_{e}^{(\beta)}(\cos (2 \omega))\right. \\
\\
\left.+j \cdot \sin (\omega) \cdot P_{o}^{(\beta)}(\cos (2 \omega))\right\}-e^{-j 2 \omega N} \\
=\frac{1}{2} e^{-j 2 \omega N}\left\{e^{-j 2 \omega N_{\Delta}} P^{(\beta)}\left(e^{j \omega}\right)-1\right\}, \quad \omega \in[0, \pi / 2]
\end{aligned}
$$

where

$$
\begin{aligned}
P^{(\beta)}\left(e^{j \omega}\right)=\{ & \cos (\omega) \cdot P_{e}^{(\beta)}(\cos (2 \omega)) \\
& \left.+j \cdot \sin (\omega) \cdot P_{o}^{(\beta)}(\cos (2 \omega))\right\}
\end{aligned}
$$

and

$$
N_{\Delta}=\left(N_{\beta} / 2\right)-N
$$

As mentioned earlier, the required delay $N$ is smaller than the length of the function $\beta(z), N_{\beta}$. The constant $N_{\Delta}$ is therefore greater than zero, and the desired response of $P^{(\beta)}\left(e^{j \omega}\right)$ is given by

$$
P_{d}^{(\beta)}\left(e^{j \omega}\right)=e^{j 2 \omega N_{\Delta}} .
$$

Equation (3.9) is recognized as a complex polynomial approximation problem. Such a complex Chebyshev or minimax approximation problem has previously been addressed by several authors [22]-[27]. Without loss of generality, we adopted the method in [25] because it is relatively simple to implement. Basically, $P_{e}^{(\beta)}(\cos (2 \omega))$ and $P_{o}^{(\beta)}(\cos (2 \omega))$ are chosen to minimize the real and imaginary parts of $P_{d}^{(\beta)}\left(e^{j \omega}\right)$ separately using the Remez exchange algorithm. In other words, the complex Chebyshev approximation is solved using two independent real Chebyshev approximations. The desired functions of $P_{e}^{(\beta)}(\cos (2 \omega))$ and $P_{o}^{(\beta)}(\cos (2 \omega))$ are then given by

and

$$
\hat{P}_{e}^{(\beta)}(\cos (2 \omega))=\frac{\cos \left(2 \omega N_{\Delta}\right)}{\cos (\omega)}
$$

$$
\hat{P}_{o}^{(\beta)}(\cos (2 \omega))=\frac{\sin \left(2 \omega N_{\Delta}\right)}{\sin (\omega)}, \quad 0<\omega<\pi / 2 .
$$

Writing $x=\cos (2 \omega)$, the two Chebyshev approximation problems can be written as

$$
\begin{aligned}
a_{k, o p t} & =\arg \min _{a_{k}} \max \left|W_{e}(x)\left(P_{e}^{(\beta)}(x)-\hat{P}_{e}^{(\beta)}(x)\right)\right| \\
b_{k, o p t} & =\arg \min _{b_{k}} \max \left|W_{o}(x)\left(P_{o}^{(\beta)}(x)-\hat{P}_{o}^{(\beta)}(x)\right)\right| \\
I_{x} & =\left(-1, \tilde{x}_{s}\right] \cup\left[x_{s}, 1\right]
\end{aligned}
$$

and

where

$$
\begin{aligned}
\tilde{x}_{s}<x_{s} & =\cos \left(2 \omega_{p}\right) \\
W_{e}(x) & =\cos (0.5 \cdot \arccos (x))
\end{aligned}
$$

and

$$
W_{o}(x)=\sin (0.5 \cdot \arccos (x)) .
$$

The interval $\left(0, \tilde{x}_{s}\right]$ is an optional disjoint interval used to control the values of $P_{e}^{(\beta)}(\cos (2 \omega))$ and $P_{o}^{(\beta)}(\cos (2 \omega))$ in the transition band of $H_{0}\left(e^{j \omega}\right)$. Since $\beta_{e}\left(e^{j \omega}\right)$ is a type-II linearphase function, it is equal to zero at $\omega=\pi$, i.e., $\beta_{e}\left(e^{j \pi}\right)=0$. On the other hand, $\beta_{o}\left(e^{j \omega}\right)$ is equal to zero at $\omega=0$, i.e., $\beta_{o}\left(e^{j 0}\right)=$ 0 , because it is a type-IV linear-phase function. The actual value of $\tilde{x}_{s}$ determines how large the values of $P_{e}^{(\beta)}(\cos (2 \omega))$ or $P_{o}^{(\beta)}(\cos (2 \omega))$ are in the interval $\left(-1, \tilde{x}_{s}\right.$ ] [i.e., in the transition band of $H_{0}\left(e^{j \omega}\right)$ ]. Equation (3.11) can readily be solved using the function REMEZ in the signal processing Toolbox of MATLAB.

$H_{0}\left(e^{j \omega}\right)$ can also be determined using the least squares design criterion. In fact, the weighted least squares error in approximating $H_{d}^{(0)}\left(e^{j \omega}\right)$ by $H_{0}\left(e^{j \omega}\right)$ is given by

$$
D_{2}=\frac{2}{\pi} \int_{0}^{\pi / 2} W_{2}(\omega)\left|E_{0}(\omega)\right|^{2} d \omega
$$

where $W_{2}(\omega)$ is a positive weighting function. Substituting (3.7) into (3.12), one gets, after some manipulation

$$
D_{2}=\boldsymbol{a}^{T} \boldsymbol{Q}_{e} \boldsymbol{a}+\boldsymbol{b}^{T} \boldsymbol{Q}_{o} \boldsymbol{b}-2 \boldsymbol{a}^{T} \boldsymbol{r}_{e}-2 \boldsymbol{b}^{T} \boldsymbol{r}_{o}+c
$$

where

$$
\begin{aligned}
\boldsymbol{a}= & \left\lfloor\begin{array}{llll}
a_{0} & a_{1} & \cdots & a_{L_{\beta}}
\end{array}\right\rfloor^{T} \\
\boldsymbol{b}= & \left\lfloor\begin{array}{llll}
b_{0} & b_{1} & \cdots & b_{L_{\beta}}
\end{array}\right\rfloor^{T} \\
\boldsymbol{r}_{\ell}= & \left\lfloor\begin{array}{llll}
r_{0}^{(\ell)} & r_{1}^{(\ell)} & \cdots & r_{L_{\beta}}^{(\ell)}
\end{array}\right], \quad \ell=o, e \\
{\left[\boldsymbol{Q}_{e}\right]_{k_{1} k_{2}}=} & \frac{2}{\pi} \int_{0}^{\pi / 2} W_{2}(\omega) \cos ^{2}(\omega) \cdot(\cos (2 \omega))^{k_{1}+k_{2}} d \omega \\
{\left[\boldsymbol{Q}_{o}\right]_{k_{1} k_{2}}=} & \frac{2}{\pi} \int_{0}^{\pi / 2} W_{2}(\omega) \sin ^{2}(\omega) \cdot(\cos (2 \omega))^{k_{1}+k_{2}} d \omega \\
r_{k}^{(e)}= & \frac{2}{\pi} \int_{0}^{\pi / 2} 2 W_{2}(\omega) \cos \left(2 \omega N_{\Delta}\right) \cdot \cos (\omega) \\
& \cdot(\cos (2 \omega))^{k} d \omega \\
r_{k}^{(o)}= & \frac{2}{\pi} \int_{0}^{\pi / 2} 2 W_{2}(\omega) \sin \left(2 \omega N_{\Delta}\right) \cdot \sin (\omega) \\
& \cdot(\cos (2 \omega))^{k} d \omega \\
c= & \frac{2}{\pi} \int_{0}^{\pi / 2} W_{2}(\omega) d \omega .
\end{aligned}
$$



by

The optimal weighted least squares solution is therefore given

$$
\boldsymbol{a}_{o p t}=\boldsymbol{Q}_{e}^{-1} \boldsymbol{r}_{e} \text { and } \boldsymbol{b}_{o p t}=\boldsymbol{Q}_{o}^{-1} \boldsymbol{r}_{o}
$$

To avoid calculating the integrals analytically, one can approximate the integral by a summation with sufficient large number of terms.

\section{B. Design of Highpass Filter}

Having assumed that $H_{0}(z)$ is a reasonably good lowpass filter, we now proceed to formulate the problem of designing $H_{1}(z)$ with $\alpha(z)$ as an even-length FIR function. It can be seen from (2.8) and (2.9) that the frequency response of $H_{1}(z)$ is dependent on both the lowpass filter $H_{0}(z)$ and the function $\alpha(z)$. The ideal frequency response of $H_{1}\left(e^{j \omega}\right)$ is

$$
H_{d}^{(1)}\left(e^{j \omega}\right)=\left\{\begin{array}{ll}
0, & 0 \leq \omega \leq \omega_{p} \\
e^{-j \omega(2 M+1)}, & \omega_{s} \leq \omega \leq \pi
\end{array} .\right.
$$

The error function $E_{1}(\omega)$ of the highpass filter $H_{1}(z)$ is defined as

$$
E_{1}(\omega)=e^{-j \omega(2 M+1)}-\alpha\left(e^{2 j \omega}\right) H_{0}\left(e^{j \omega}\right)-H_{d}^{(1)}\left(e^{j \omega}\right)
$$

Since the minimization in (3.16) involves $\alpha\left(e^{2 j \omega}\right)$, which is periodic with period $\pi$, it is different from the conventional Chebyshev approximation. In fact, for a given value of $\omega \in[0, \pi / 2], \alpha\left(e^{2 j \omega}\right)$ will affect the values of $H_{1}\left(e^{j \omega}\right)$ at $\omega$ as well as $\omega+\pi$. Fortunately, it is observed that the magnitude of $\alpha\left(e^{2 j \omega}\right)$ is almost equal to one, except around $\omega=\pi / 2$, where it is even smaller. It then follows from (2.8) that the passband ripple of $H_{1}\left(e^{j \omega}\right)$ is approximately equal to the stopband error of $H_{0}\left(e^{j \omega}\right)$. This allows us to minimize only the stopband attenuation of $H_{1}\left(e^{j \omega}\right)$ using $\alpha\left(e^{2 j \omega}\right)$ instead of minimizing (3.16) over the passband and stopband and relies on the high stopband attenuation of $H_{0}\left(e^{j \omega}\right)$ to achieve small passband ripple. First of all, let us consider the case where $\alpha(z)$ is an even-length FIR filter with length $N_{\alpha}$. The odd value case can be derived similarly and summarized in Appendix A. Using again the even and odd parts decomposition of the impulse response $h_{\alpha}(n)$ of $\alpha(z)$, we have

$$
h_{\alpha}(n)=h_{\alpha, e}(n)+h_{\alpha, o}(n)
$$

where

$$
\begin{aligned}
& h_{\alpha, e}(n)=\frac{h_{\alpha}(n)+h_{\alpha}\left(N_{\alpha}-n-1\right)}{2} \\
& h_{\alpha, o}(n)=\frac{h_{\alpha}(n)-h_{\alpha}\left(N_{\alpha}-n-1\right)}{2} .
\end{aligned}
$$

Similar to $\beta_{e}(z)$ and $\beta_{o}(z), \alpha_{e}(z)$ and $\alpha_{o}(z)$, which are the $z$-transforms of $h_{\alpha, e}(n)$ and $h_{\alpha, o}(n)$, are type-II and type-IV linear-phase FIR functions of length $N_{\alpha}$, respectively. $\alpha_{e}\left(e^{j \omega}\right)$ and $\alpha_{o}\left(e^{j \omega}\right)$ can therefore be expressed as follows:

$$
\begin{aligned}
& \alpha_{e}\left(e^{j \omega}\right)=e^{-j \omega\left(N_{\alpha}-1\right) / 2} \cos (\omega / 2) P_{e}^{(\alpha)}(\cos (\omega)) \\
& \alpha_{o}\left(e^{j \omega}\right)=j e^{-j \omega\left(N_{\alpha}-1\right) / 2} \sin (\omega / 2) P_{o}^{(\alpha)}(\cos (\omega))
\end{aligned}
$$

where

$$
\begin{aligned}
& P_{e}^{(\alpha)}(\cos (\omega))=\sum_{k=0}^{L_{\alpha}} \tilde{a}_{k}(\cos (\omega))^{k} \\
& P_{o}^{(\alpha)}(\cos (\omega))=\sum_{k=0}^{L_{\alpha}} \tilde{b}_{k}(\cos (\omega))^{k} .
\end{aligned}
$$

$L_{\alpha}$ is the order of the polynomials and is equal to $\left(N_{\alpha} / 2\right)-1$. Substituting (3.19) and (3.17) into (3.16), we have

$$
\begin{gathered}
E_{1}(\omega)=e^{-j \omega(2 M+1)}-e^{-j \omega\left(N_{\alpha}-1\right)} P^{(\alpha)}\left(e^{j \omega}\right) H_{0}\left(e^{j \omega}\right) \\
\omega \in[0, \pi / 2]
\end{gathered}
$$

where

$$
\begin{aligned}
P^{(\alpha)}\left(e^{j \omega}\right)=\{ & \cos (\omega) \cdot P_{e}^{(\alpha)}(\cos (2 \omega)) \\
& \left.+j \cdot \sin (\omega) \cdot P_{o}^{(\alpha)}(\cos (2 \omega))\right\} .
\end{aligned}
$$

Let the lowpass filter $H_{0}\left(e^{j \omega}\right)$ be written as

$$
H_{0}\left(e^{j \omega}\right)=A\left(e^{j \omega}\right) e^{-j 2 \omega N}
$$

where $A\left(e^{j \omega}\right)$ is a complex function, and it is approximately equal to one in the passband and 0 otherwise when the order of $\beta(\omega)$ is sufficiently high. Substituting (3.22) into (3.20), one obtains

$$
\begin{gathered}
E_{1}(\omega)=e^{-j \omega(2 M+1)}-e^{-j \omega\left(\left(N_{\alpha}-1\right)+2 N\right)} P^{(\alpha)}\left(e^{j \omega}\right) \cdot A\left(e^{j \omega}\right) \\
=e^{-j \omega(2 M+1)}\left\{1-e^{-j 2 \omega M_{\Delta}} P^{(\alpha)}\left(e^{j \omega}\right) \cdot A\left(e^{j \omega}\right)\right\} \\
\omega \in[0, \pi / 2]
\end{gathered}
$$

where $M_{\Delta}=\left(N_{\alpha} / 2\right)+(N-M)-1$. From (3.23), it can be seen that the ideal response of $P^{(\alpha)}\left(e^{j \omega}\right)$ is

$$
P_{d}^{(\alpha)}\left(e^{j \omega}\right)=e^{j \omega M_{\Delta}} A^{-1}\left(e^{j \omega}\right), \quad \omega \in[0, \pi / 2] .
$$

Again, if $P_{e}^{(\alpha)}(\cos (\omega))$ and $P_{o}^{(\alpha)}(\cos (\omega))$ are used to approximate the real and imaginary parts of $P_{d}^{(\alpha)}\left(e^{j \omega}\right)$ separately, then their ideal responses are

$$
\begin{aligned}
\hat{P}_{e}^{(\alpha)}(\cos (2 \omega)) & =\frac{\operatorname{Re}\left(A^{-1}\left(e^{j \omega}\right) e^{j 2 \omega M_{\Delta}}\right)}{\cos (\omega)} \\
& =\frac{\operatorname{Re}\left(A *\left(e^{j \omega}\right) e^{j 2 \omega M_{\Delta}}\right)}{\left|A\left(e^{j \omega}\right)\right|^{2} \cos (\omega)} \\
\hat{P}_{o}^{(\alpha)}(\cos (2 \omega)) & =\frac{\operatorname{Im}\left(A^{-1}\left(e^{j \omega}\right) e^{j 2 \omega M_{\Delta}}\right)}{\sin (\omega)} \\
& =\frac{\operatorname{Im}\left(A *\left(e^{j \omega}\right) e^{j 2 \omega M_{\Delta}}\right)}{\left|A\left(e^{j \omega}\right)\right|^{2} \sin (\omega)}, \quad 0<\omega<\pi / 2 .
\end{aligned}
$$

Writing $x=\cos (2 \omega)$, the minimax design problem can be written as

$$
\begin{aligned}
& \tilde{a}_{k, \text { opt }}=\arg \min _{a_{k}} \max \left|\tilde{W}_{e}(x)\left(P_{e}^{(\alpha)}(x)-\hat{P}_{e}^{(\alpha)}(x)\right)\right| \\
& \tilde{b}_{k, \text { opt }}=\arg \min _{b_{k}} \max \left|\tilde{W}_{o}(x)\left(P_{o}^{(\alpha)}(x)-\hat{P}_{o}^{(\alpha)}(x)\right)\right|
\end{aligned}
$$


where

$$
\begin{aligned}
I_{x} & =\left(-1, \tilde{x}_{s}\right] \cup\left[x_{s}, 1\right], \quad \tilde{x}_{s}<x_{s}=\cos \left(2 \omega_{p}\right) \\
\tilde{W}_{e}(x) & =\cos (0.5 \cdot \arccos (x))\left|A\left(e^{j 0.5 \arccos (x)}\right)\right|^{2} \\
\tilde{W}_{o}(x) & =\sin (0.5 \cdot \arccos (x))\left|A\left(e^{j 0.5 \arccos (x)}\right)\right|^{2} .
\end{aligned}
$$

The two Chebyshev approximation problems in (3.26) are similar to (3.11), which again can be solved by the Remez exchange algorithm. If the least squares criterion is used, it can be shown, as in Section III-A, that the optimal values of $\tilde{a}_{k}$ and $\tilde{b}_{k}$ are given by

$$
\tilde{\boldsymbol{a}}_{o p t}=\tilde{\boldsymbol{Q}}_{e}^{-1} \tilde{\boldsymbol{r}}_{e} \text { and } \tilde{\boldsymbol{b}}_{o p t}=-\tilde{\boldsymbol{Q}}_{o}^{-1} \tilde{\boldsymbol{r}}_{o}
$$

where

$$
\begin{aligned}
& \tilde{\boldsymbol{a}}=\left[\begin{array}{llll}
\tilde{a}_{0} & \tilde{a}_{1} & \cdots & \tilde{a}_{L_{\alpha}}
\end{array}\right]^{T} \\
& \boldsymbol{b}=\left[\begin{array}{llll}
\tilde{b}_{0} & \tilde{b}_{1} & \cdots & \tilde{b}_{L_{\alpha}}
\end{array}\right]^{T} \\
& \tilde{\boldsymbol{r}}_{\ell}=\left[\begin{array}{llll}
\tilde{r}_{0}^{(\ell)} & \tilde{r}_{1}^{(\ell)} & \ldots & \tilde{r}_{L_{\alpha}}^{(\ell)}
\end{array}\right]^{T}, \quad \ell=o, e \\
& {\left[\tilde{\boldsymbol{Q}}_{e}\right]_{k_{1} k_{2}}=\frac{2}{\pi} \int_{0}^{\pi / 2} \tilde{W}_{2}(\omega) \cos ^{2}(\omega)} \\
& \cdot(\cos (2 \omega))^{k_{1}+k_{2}}\left|A\left(e^{j \omega}\right)\right|^{2} d \omega \\
& {\left[\tilde{\boldsymbol{Q}}_{o}\right]_{k_{1} k_{2}}=\frac{2}{\pi} \int_{0}^{\pi / 2} \tilde{W}_{2}(\omega) \sin ^{2}(\omega)} \\
& \cdot(\cos (2 \omega))^{k_{1}+k_{2}}\left|A\left(e^{j \omega}\right)\right|^{2} d \omega \\
& \tilde{r}_{k}^{(e)}=\frac{2}{\pi} \int_{0}^{\pi / 2} 2 \tilde{W}_{2}(\omega) \operatorname{Re}\left[A\left(e^{j \omega}\right) e^{-j 2 \omega M_{\Delta}}\right] \\
& \cdot\left(\cos (\omega) \cdot(\cos (2 \omega))^{k}\right) d \omega \\
& \tilde{r}_{k}^{(o)}=\frac{2}{\pi} \int_{0}^{\pi / 2} 2 \tilde{W}_{2}(\omega) \operatorname{Im}\left[A\left(e^{j \omega}\right) e^{-j 2 \omega M_{\Delta}}\right] \\
& \cdot\left(\sin (\omega) \cdot(\cos (2 \omega))^{k}\right) d \omega \\
& c=\frac{2}{\pi} \int_{0}^{\pi / 2} \tilde{W}_{2}(\omega) d \omega .
\end{aligned}
$$

It can be seen that if $N_{\Delta}=M_{\Delta}=0$, the filterbank becomes linear-phase. Therefore, all the above results are also applicable to linear-phase FIR filterbanks. It should also be noted that (3.25) and (3.27) are valid if $H_{0}\left(e^{j \omega}\right)$ is an IIR filter such as the allpass based filter [9]. This property has been utilized to design a class of IIR filterbanks with $\beta(z)$ and $\alpha(z)$ chosen as allpass and linear-phase functions, respectively [31]. Because the proposed low-delay filterbank is derived from the structure in Fig. 2, it is by no means complete and optimal. For example, it is possible to express any two-channel PR FIR filterbank using the lifting scheme. As mentioned earlier, this will, in general, lead to an unconstrained optimization of highly nonlinear function, which is more difficult to solve. In Section III-E, it is demonstrated by several design examples that low-delay PR filterbanks with very good frequency characteristics can still be obtained by the proposed structure with very low design and implementation complexities.

\section{Combined Weighting Function}

As mentioned earlier in Section III-A, solving the complex Chebyshev approximation problem using two independent real Chebyshev problems of its real and imaginary parts is inherently suboptimal. The loss in stopband attenuation can be as high as $3 \mathrm{~dB}$ [24]. Better results can be achieved by using the method in [24]. In this paper, a simple reweight technique is employed to improve the performance of the independent approach in [25]. More precisely, the errors in approximating the real and imaginary parts with the independent approach is used to reweight the original weighting function to achieve an overall equiripple complex error.

Let $\delta_{e}(\omega)$ and $\delta_{o}(\omega)$ be, respectively, the approximation error of the two independent Chebyshev approximations. The complex approximation error $\delta(\omega)$ is

$$
\delta(\omega)=\delta_{e}(\omega)+j \delta_{o}(\omega)
$$

and its norm is given by $|\delta(\omega)|=\sqrt{\delta_{e}^{2}(\omega)+\delta_{o}^{2}(\omega)}$. It can be seen that although $\delta_{e}(\omega)$ and $\delta_{o}(\omega)$ are equiripple, $|\delta(\omega)|$ is not necessarily equiripple. The reweight technique used here modifies the original weighting function $W(\omega)$ as follows:

$$
W_{\delta}(\omega)=W(\omega)|\delta(\omega)|^{P}
$$

where $P$ is a positive integer. From experimental results, it was found that $P=2$ gave better performance than $P=1$. $|\delta(\omega)|$ can be estimated by performing an initial independent Chebyshev approximation. After that, the problem is solved again using the new weighting function $W_{\delta}(\omega)$ instead of $W(\omega)$. It is found that this method can provide considerable improvement over the independent Chebyshev approximations in [25]. In this paper, all the complex Chebyshev approximations involving $\beta(z)$ and $\alpha(z)$ are assumed to be carried out by this method.

\section{Selection of $N, M, N_{\beta}$ and $N_{\alpha}$}

In practical applications of such a low-delay FIR filterbank, one frequently encountered problem is the following: Given $\delta_{s_{0}}$ and $\delta_{s_{1}}$, the stopband attenuation of $H_{0}(z)$ and $H_{1}(z)$, and their cutoff frequencies $\omega_{p_{0}}, \omega_{s_{0}}$ and $\omega_{p_{1}}, \omega_{s_{1}}$, how are the delay parameters $N$ and $M$ and the lengths $N_{\beta}$ and $N_{\alpha}$ chosen? Here, some design guidelines regarding the selection of such parameters will be given. The linear-phase and nonlinear-phase cases with $\omega_{p_{0}}=\omega_{s_{1}}=\omega_{p}$ and $\omega_{s_{0}}=\omega_{p_{1}}=\omega_{s}$ are discussed in turn.

Linear-Phase Case: In this case, both $\beta(z)$ and $\alpha(z)$ are linear-phase. It is observed that $H_{0}(z)$ is a linear-phase halfband filter with identical passband and stopband ripples. The formula proposed by Kaiser (in [38, eq. (7.104)]) can therefore be used to estimate $L_{0}$ (the order of this halfband filter) and the length of $\beta(z)$.

$$
L_{0}=\frac{-20 \log _{10} \delta-13}{2.324 \Delta \omega}
$$

where $\Delta \omega=\omega_{s}-\omega_{p}$, and $\delta=\delta_{p_{0}}=\delta_{s_{0}}$. The length of $\beta(z)$, $N_{\beta}$ is therefore approximately given by $\left(L_{0}+2\right) / 2$. To estimate $N_{\alpha}$ (the length of $\alpha(z)$ ), it is observed that when $N_{\alpha}$ is identical to $N_{\beta}$, the stopband attenuation of $H_{1}(z)$ is about $10 \mathrm{~dB}$ lower 

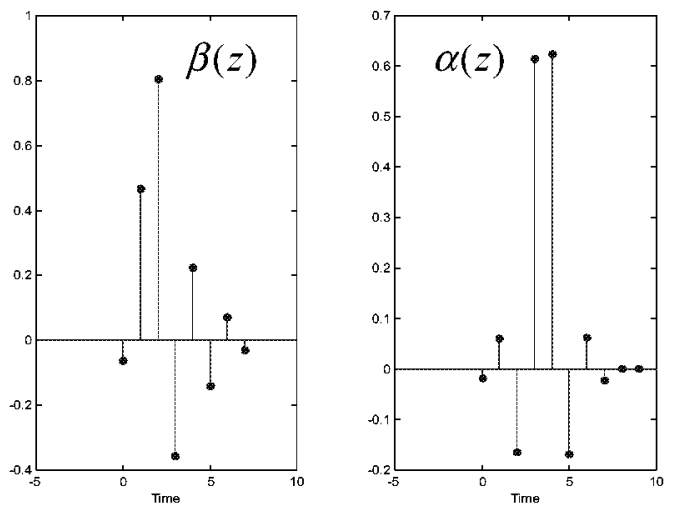

(a)

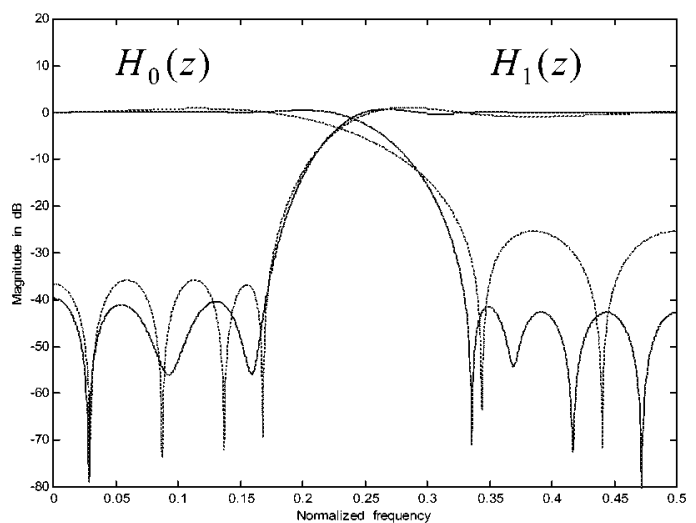

(c)

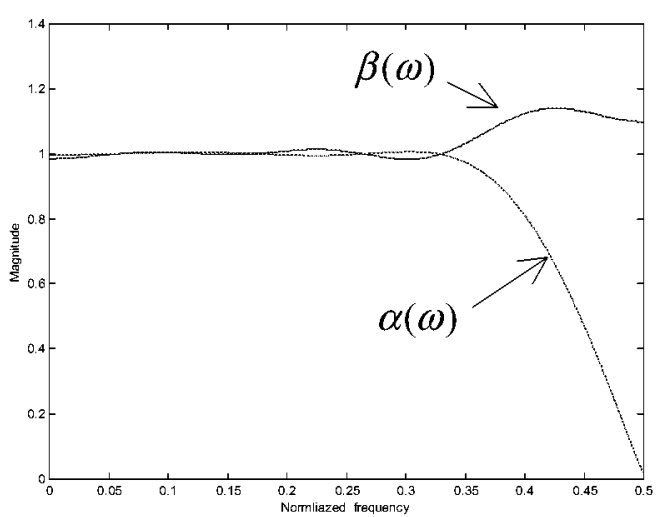

(b)

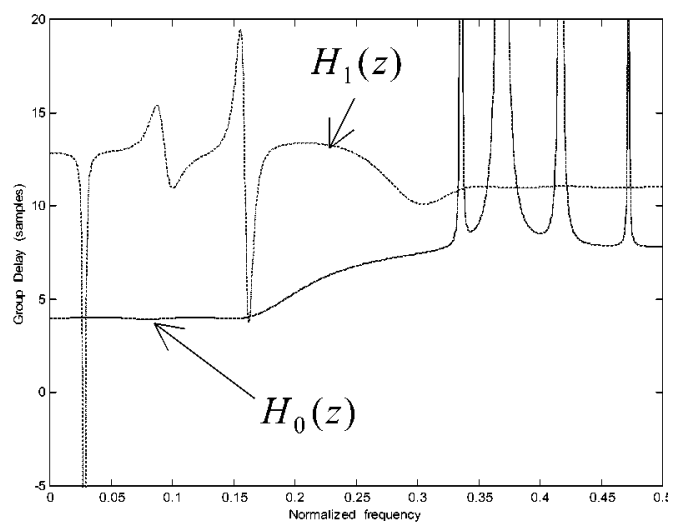

(d)

Fig. 3. Low delay analysis filters in Example 3.1 designed by the Remez exchange algorithm. (a) Impulse responses of $\beta(z)$ and $\alpha(z)$. (b) Magnitude responses of $\beta(z)$ and $\alpha(z)$. (c) Magnitude responses of low delay analysis filters $H_{0}(z)$ and $H_{1}(z)$ (solid lines) and their linear-phase counterparts (dashed lines). (d) Group delay responses of low delay analysis filters.

than that of $H_{0}(z)$. To achieve similar stopband attenuation for $H_{0}(z)$ and $H_{1}(z)$, the length of $\alpha(z)$ can be slightly increased at the expense of higher system delay.

Nonlinear-Phase Case: If $\beta(z)$ is nonlinear-phase, the stopband attenuation of $H_{0}(z)$ is $1-5 \mathrm{~dB}$ lower than that of a linearphase halfband filter with the same length, depending on the passband delay. Normally, the passband delay of $H_{0}(z)$ can be reduced by a factor of two, as compared with the linear-phase halfband filter without too much degradation in stopband attenuation. However, significant bumping $(>1 \mathrm{~dB})$ will appear in the transition band of $H_{0}(z)$ if the passband delay is lowered further. This observation allows us to estimate the length of $\beta(z)$ using its linear-phase counterpart as a reference, which in turn can be estimated using the formula in (3.30). To estimate $N_{\alpha}$, it is observed that when $M$ is equal to $3 N-1$, the stopband attenuation of $H_{1}(z)$ is comparable with that of $H_{0}(z)$. With these choices (together with the one mentioned in linear-phase case), the total system delay of the filterbank is only two thirds of its linear-phase counterpart with comparable stopband atteunation. Slightly higher stopband attenuation can be obtained when the system delay is increased by increasing $N$ and $M$. Let us consider Example 3.1. If the required stopband attenuation for $H_{0}(z)$ is $40 \mathrm{~dB}$ (or $\delta_{s_{0}} \leq 0.01$ ) and the cut-off fre- quencies are $\omega_{p}=0.34 \pi$ and $\omega_{s}=0.66 \pi$, the order of the linear-phase filter $H_{0}(z)$, as suggested by (3.30), is $L_{0}=12$. Because the linear-phase $\beta(z)$ is a type-II FIR filter, the length of $\beta(z)$ should be $N_{\beta}=8$. The length of the linear-phase filter $\alpha(z)$ should be slightly larger than $N_{\beta}$ to achieve a comparable stopband attenuation for $H_{0}(z)$ and $H_{1}(z)$. In this example, it is found that $N_{\alpha}=10$ is sufficient. For the low-delay filterbank, the passband delay of $H_{0}(z)$, as discussed earlier, can be chosen as one half of its linear-phase counterpart. The delay parameter $N$ is therefore chosen as 2 . The remaining delay parameter $M$ can be chosen as $3 N-1$, which is equal to 5 . The system delay of the resulting low-delay filterbank is only 15 samples. Note that a linear-phase filterbank, using the same structure, will require a system delay of 23 samples to achieve the same stopband attenuation. The same procedure can be applied to example 3.2, except that $N_{\beta}$ and $N_{\alpha}$ are now odd numbers.

\section{E. Design Examples}

In this section, the proposed method is evaluated and compared with other conventional methods through several design examples.

Example 3.1: In this design example, $\beta(z)$ and $\alpha(z)$ are nonlinear-phase FIR functions with lengths $N_{\beta}=8$ and $N_{\alpha}=$ 
TABLE I

COEFFICIENTS OF $\beta(z)$ AND $\alpha(z)$ IN EXAMPLE 3.1 AND EXAMPLE 4.1

\begin{tabular}{c|c|c|c|c}
\hline & \multicolumn{2}{|c|}{ Example 3.1} & \multicolumn{2}{c}{ Example 4.1} \\
\hline $\mathrm{n}$ & $\beta(z)$ & $\alpha(z)$ & $\beta(z)$ & $\alpha(z)$ \\
\hline 0 & $-6.122981893326920 \mathrm{e}-002$ & $-1.743263074129322 \mathrm{e}-002$ & $-6.860333316293123 \mathrm{e}-002$ & $-2.231767854809924 \mathrm{e}-002$ \\
\hline 1 & $4.665091805090530 \mathrm{e}-001$ & $6.148674113264712 \mathrm{e}-002$ & $4.852634764782267 \mathrm{e}-001$ & $7.354326492786681 \mathrm{e}-002$ \\
\hline 2 & $8.041133592808363 \mathrm{e}-001$ & $-1.642674206548875 \mathrm{e}-001$ & $7.874089332511693 \mathrm{e}-001$ & $-1.719970076192219 \mathrm{e}-001$ \\
\hline 3 & $-3.537827046312050 \mathrm{e}-001$ & $6.149753727946287 \mathrm{e}-001$ & $-3.325236490599400 \mathrm{e}-001$ & $6.210882208958493 \mathrm{e}-001$ \\
\hline 4 & $2.255496470471235 \mathrm{e}-001$ & $6.255681859248121 \mathrm{e}-001$ & $2.147668758288946 \mathrm{e}-001$ & $6.249609215611015 \mathrm{e}-001$ \\
\hline 5 & $-1.391949022596057 \mathrm{e}-001$ & $-1.679968084966865 \mathrm{e}-001$ & $-1.314446186132179 \mathrm{e}-001$ & $-1.764457687368829 \mathrm{e}-001$ \\
\hline 6 & $7.365179626980009 \mathrm{e}-002$ & $6.298270160800767 \mathrm{e}-002$ & $8.009306132427543 \mathrm{e}-002$ & $7.459638702347382 \mathrm{e}-002$ \\
\hline 7 & $-3.016262583933727 \mathrm{e}-002$ & $-2.117141328591070 \mathrm{e}-002$ & $-3.496074604647677 \mathrm{e}-002$ & $-3.006253412136717 \mathrm{e}-002$ \\
\hline 8 & & $1.197988381898378 \mathrm{e}-003$ & & $6.759899558032634 \mathrm{e}-003$ \\
\hline 9 & & $1.565945242569830 \mathrm{e}-003$ & & $-1.257049407528445 \mathrm{e}-004$ \\
\hline
\end{tabular}

10. The orders of the polynomials $P_{e}^{(\beta)}(x)$ and $P_{o}^{(\beta)}(x)$ are $L_{\beta}=3$, and the orders of $P_{e}^{(\alpha)}(x)$ and $P_{o}^{(\alpha)}(x)$ are $L_{\alpha}=$ 4. The delay parameters $N$ and $M$ are, respectively, 2 and 5. The overall system delay is $n_{0}=15$ samples. Fig. 3(a) plots the impulse responses of $\beta(z)$ and $\alpha(z)$ designed by the proposed method using the Remez exchange algorithm. Their coefficients are listed in Table I. Fig. 3(b) displays the magnitude responses of $\beta(z)$ (solid line) and $\alpha(z)$ (dashed line). To demonstrate the higher stopband attenuation of the proposed low-delay FIR filterbanks over their linear-phase counterpart, a two-channel linear-phase FIR filterbanks with the same system delay was designed by the proposed algorithm. The delay parameters $N$ and $M$ are still 2 and 5, but the lengths of $\beta(z)$ and $\alpha(z)$ are shortened to $N_{\beta}=4$ and $N_{\alpha}=8$, respectively, due to the linear-phase constraint. Fig. 3(c) displays the magnitude responses of the low-delay analysis filters $H_{0}(z)$ and $H_{1}(z)$ (solid lines), whereas those of the linear-phase analysis filters are plotted in dashed lines. It is observed that under the same system delay $\left(n_{0}=15\right)$ and the same passband and stopband cut-off frequencies $\left(\omega_{p}=0.34 \pi\right.$ and $\left.\omega_{s}=0.66 \pi\right)$, the stopband attenuation of the proposed low-delay lowpass analysis filter is much higher than its linear-phase counterpart (42 $d B$ versus $26 \mathrm{~dB}$ ). On the other hand, the stopband attenuation of the low-delay highpass analysis filter is slightly higher than its linear-phase counterpart ( $40 \mathrm{~dB}$ versus $36 \mathrm{~dB}$ ). This improvement in the stopband attenuation, however, will require additional arithmetic computations in filterbank implementation [the number of the variables of $\beta(z)$ and $\alpha(z)$ in the low-delay case is 18 , whereas that of the linear-phase is only 6]. To reduce the implementation complexity of the proposed low-delay filterbanks, a very efficient multiplier-less realization of the proposed low-delay filterbanks using the SOPOT coefficients is proposed later in Section V. Fig. 3(d) plots the group delay responses of the low-delay analysis filters $H_{0}(z)$ (solid line) and $H_{1}(z)$ (dashed lines), respectively. It can also be seen from Fig. 3(d) that both $H_{0}(z)$ and $H_{1}(z)$ are approximately linear-phase in their passbands.

In order to compare the proposed method with the conventional low-delay design methods in [10] and [16], the cutoff fre- quencies and system delay are identical to the design examples in [10, Fig. 5] and [16, Fig. 3]. The stopband attenuation of the analysis filters in [10] is about $39 \mathrm{~dB}$. The stopband attenuation of $H_{0}(z)$ and $H_{1}(z)$ in [16, Fig. 3] are, respectively, $45 \mathrm{~dB}$ and $40 \mathrm{~dB}$. Therefore, the proposed analysis filters have comparable passband and stopband performance as those in [10] and [16]. By adjusting the cutoff frequencies to $\omega_{p}=0.24 \pi$ and $\omega_{s}=0.76 \pi$ and $\omega_{p}=0.4 \pi$ and $\omega_{s}=0.6 \pi$, the proposed method can still provide comparable stopband attenuation as that in [14, Fig. 1] ( $55 \mathrm{~dB}$ versus $56 \mathrm{~dB})$ and a higher stopband attenuation than that in [20, Fig. 2] (30 dB versus 25 $\mathrm{dB})$. It should be noted that due to the simplified structure of the proposed filterbank, its design complexity is very low, and there is no reconstruction error, which is always present in other methods based on constrained nonlinear optimization [10], [20].

Furthermore, as mentioned earlier, the proposed filterbank structure is still PR even under coefficient quantization, unlike the direct form in [16]. The design complexity of the proposed method is also much lower than the unconstrained nonlinear optimization methods in [12] and [14], thanks to the Remez exchange algorithm. The overall performance comparison between the proposed method and the conventional low-delay filterbank design methods [10], [14], [16], [20] is summarized in Table II, where the arithmetic complexity is simply defined as the number of multiplications and additions in implementing the filterbank. This demonstrates the good performance, flexibility, low implementation, and design complexities of the proposed method, as compared with conventional methods. Fig. 4 displays the magnitude and group delay responses of the low-delay analysis filters $H_{0}(z)$ and $H_{1}(z)$ designed by the proposed least squares method. It can be observed that the stopband attenuations of $H_{0}(z)$ and $H_{1}(z)$ are comparable to the minimax design in Fig. 3, but the stopband attenuation of $H_{0}(z)$ and $H_{1}(z)$ around $\omega=0$ or $\omega=\pi$ is slightly higher in the least squares case.

Example 3.2: In this example, a two-channel low-delay FIR filterbank with odd filter length is designed. The lengths of $\beta(z)$ and $\alpha(z)$ are $N_{\beta}=13$ and $N_{\alpha}=15$, respectively. The orders of the corresponding Chebyshev polynomials $P_{e}^{(\beta)}(x)$ and 
TABLE II

Overall Performance of Example 3.1 Compared with Other CONVENTIONAl Methods. Note that $\omega_{p}$. Is the Passband CutofF FreQuency OF

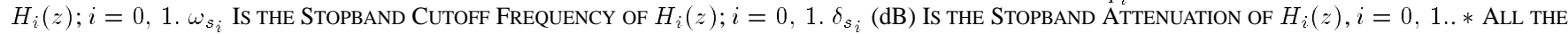
Systems Have 15 Samples Overall Delay. $* *$ The Cutoff Frequencies Have BeEN AdJusted to Be Comparable With ThOSE OF [14] OR [20]. $* * *$ NUMBer OF MULTIPLICATIONS AND ADDITIONS PER SAMPLE (ANALYSIS FilTERS)

\begin{tabular}{|c|c|c|c|c|c|c|c|c|c|}
\hline & \multicolumn{5}{|c|}{ Ex. 3.1 vs. Linear Phase, Ref. $[10,16]$ and Ex. 5.1} & \multicolumn{2}{|c|}{ Ex. 3.1 vs. Ref. $[14]$} & \multicolumn{2}{|c|}{ Ex. 3.1 vs. Ref. [20] } \\
\hline & Ex. 3.1 & LP & {$[10]$} & {$[16]$} & Ex. 5.1 & Ex. $3.1 * *$ & {$[14]$} & Ex. $3.1^{* *}$ & {$[20]$} \\
\hline$\omega_{p_{0}}$ & $0.34 \pi$ & $0.34 \pi$ & $0.38 \pi$ & $0.4 \pi$ & $0.34 \pi$ & $0.24 \pi$ & $0.3 \pi$ & $0.4 \pi$ & $0.4 \pi$ \\
\hline$\omega_{s_{0}}$ & $0.66 \pi$ & $0.66 \pi$ & $0.68 \pi$ & $0.7 \pi$ & $0.66 \pi$ & $0.76 \pi$ & $0.8 \pi$ & $0.6 \pi$ & $0.6 \pi$ \\
\hline$\omega_{p_{1}}$ & $0.66 \pi$ & $0.66 \pi$ & $0.68 \pi$ & $0.6 \pi$ & $0.66 \pi$ & $0.76 \pi$ & $0.7 \pi$ & $0.6 \pi$ & $0.6 \pi$ \\
\hline$\omega_{s_{1}}$ & $0.34 \pi$ & $0.34 \pi$ & $0.38 \pi$ & $0.3 \pi$ & $0.34 \pi$ & $0.24 \pi$ & $0.2 \pi$ & $0.4 \pi$ & $0.4 \pi$ \\
\hline$\delta_{s_{0}}(\mathrm{~dB})$ & 42 & 26 & 39 & 45 & 39 & 55 & 56 & 30 & 25 \\
\hline$\delta_{s_{1}}(\mathrm{~dB})$ & 40 & 36 & 39 & 40 & 40 & 54 & 57 & 29 & 25 \\
\hline PR & Yes & Yes & No & Yes & Yes & Yes & Yes & Yes & No \\
\hline $\begin{array}{l}\text { Robust to } \\
\text { quantization }\end{array}$ & Yes & Yes & No & No & Yes & Yes & Yes & Yes & No \\
\hline $\begin{array}{l}\text { Filter length } \\
\text { low/high pass }\end{array}$ & $15 / 33$ & $15 / 33$ & $16 / 16$ & $22 / 28$ & $15 / 33$ & $15 / 33$ & $32 / 32$ & $15 / 33$ & $28 / 36$ \\
\hline $\begin{array}{c}\text { Implementation } \\
\text { Complexity } \\
* * * \\
\end{array}$ & $\begin{array}{l}9 \text { Mul. } \\
9 \text { Add. }\end{array}$ & $\begin{array}{l}3 \text { Mul. } \\
6 \text { Add. }\end{array}$ & $\begin{array}{l}32 \text { Mul. } \\
30 \text { Add. }\end{array}$ & $\begin{array}{l}50 \mathrm{Mul} . \\
48 \mathrm{Add} .\end{array}$ & $\begin{array}{l}19.5 \\
\text { Add. }\end{array}$ & $\begin{array}{l}9 \text { Mul. } \\
9 \text { Add. }\end{array}$ & $\begin{array}{l}11 \text { Mul. } \\
9 \text { Add. }\end{array}$ & $\begin{array}{l}9 \text { Mul. } \\
9 \text { Add. }\end{array}$ & $\begin{array}{l}64 \text { Mul. } \\
62 \text { Add. }\end{array}$ \\
\hline $\begin{array}{c}\text { Design } \\
\text { Complexity }\end{array}$ & low & low & high & medium & very high & low & high & low & high \\
\hline
\end{tabular}

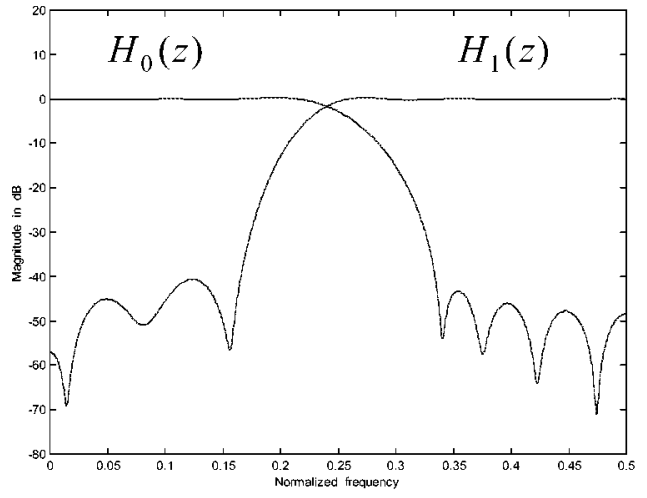

(a)

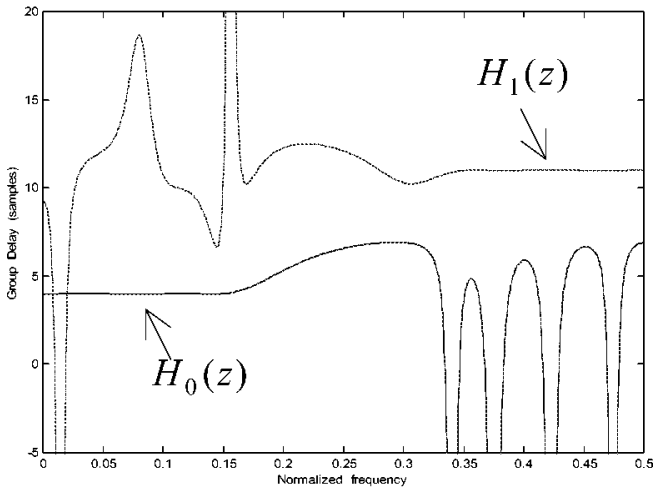

(b)

Fig. 4. Analysis filters in Example 3.1 designed by the least squares method. (a) Magnitude responses of analysis filters $H_{0}(z)$ and $H_{1}(z)$. (b) Their group delay responses.

$P_{o}^{(\beta)}(x)$ are $L_{\beta_{e}}=6$ and $L_{\beta_{o}}=5$, whereas those of $P_{e}^{(\alpha)}(x)$ and $P_{o}^{(\alpha)}(x)$ are $L_{\alpha_{e}}=7$ and $L_{\alpha_{o}}=6$. The low-delay parameters $N_{\Delta}$ and $M_{\Delta}$ are chosen to be 3.5 and 1.5, respectively. The overall system delay of the filterbank is $n_{0}=23$ samples with $N=3$ and $M=8$. Fig. 5 plots the magnitude and group delay responses of the analysis filters $H_{0}(z)$ and $H_{1}(z)$. It can be seen that the stopband attenuation of $H_{0}(z)$ and $H_{1}(z)$ is about 39 $\mathrm{dB}$, and their passband and stopband cutoff frequencies are, respectively, $\omega_{p}=0.41 \pi$ and $\omega_{s}=0.59 \pi$. The transition band is sharper than that of Example 3.1 due to the higher system delay. It is also noted that there is a bump (about $1.5 \mathrm{~dB}$ ) near the transition band of $H_{1}(z)$, which will, in general, increase as the length of $\alpha(z)$ increases for a given system delay. Detailed coefficients of the filterbanks are given in Table III.

\section{Design of Low-Delay WaVelet Bases}

The theory of wavelets is closely related to that of multirate filterbanks [28], [29]. It has been shown that discrete dyadic wavelets can be obtained from two-channel PR filterbanks with added regularity condition. For biorthogonal dyadic wavelet bases, it had been proved that [28] $H_{0}(z)$ and $G_{0}(z)$ should have $K_{0}$ (or $\tilde{K}_{0}$ ) zeros at $z=-1$ (the $K$-regularity condition). In addition, $H_{1}(z)$ and $G_{1}(z)$ should also have at least one zero at $z=1$. This is equivalent to saying that

$$
\begin{aligned}
& \left.\frac{d^{k} H_{0}(z)}{d z^{k}}\right|_{z=-1}=0, \quad k=0,1, \cdots, K_{0}-1 \\
& \left.\frac{d^{k} G_{0}(z)}{d z^{k}}\right|_{z=-1}=0, \quad k=0,1, \cdots, \tilde{K}_{0}-1 .
\end{aligned}
$$




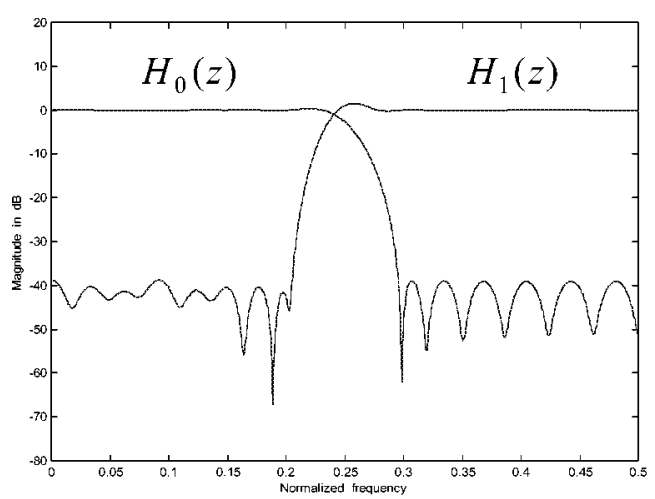

(a)

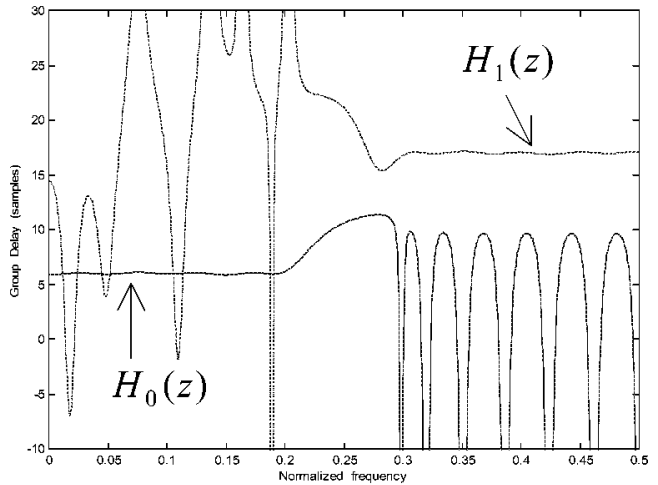

(b)

Fig. 5. Analysis filters in example 3.2 designed by the Remez exchange algorithm (a) magnitude responses of analysis filters $H_{0}(z)$ and $H_{1}(z)$; (b) their group delay responses.

TABLE III

COEFFICIENTS OF $\beta(z)$ AND $\alpha(z)$ IN EXAMPLE 3.2

\begin{tabular}{c|c|c}
\hline & \multicolumn{2}{|c}{ Example 3.2} \\
\hline $\mathrm{n}$ & $\beta(z)$ & $\alpha(z)$ \\
\hline 0 & $4.294779413338781 \mathrm{e}-002$ & $-1.163701925116906 \mathrm{e}-002$ \\
\hline 1 & $-1.170835357798205 \mathrm{e}-001$ & $2.260366411090361 \mathrm{e}-002$ \\
\hline 2 & $5.390400943595075 \mathrm{e}-001$ & $-5.288511796238420 \mathrm{e}-002$ \\
\hline 3 & $7.324070751151649 \mathrm{e}-001$ & $8.395452202030107 \mathrm{e}-002$ \\
\hline 4 & $-3.017651897513106 \mathrm{e}-001$ & $-1.800816790721913 \mathrm{e}-001$ \\
\hline 5 & $2.064998768532979 \mathrm{e}-001$ & $6.096728386449619 \mathrm{e}-001$ \\
\hline 6 & $-1.563554928168682 \mathrm{e}-001$ & $6.553300405703779 \mathrm{e}-001$ \\
\hline 7 & $1.201468774531898 \mathrm{e}-001$ & $-2.167265245937488 \mathrm{e}-001$ \\
\hline 8 & $-9.042900882382673 \mathrm{e}-002$ & $1.243710163194015 \mathrm{e}-001$ \\
\hline 9 & $6.521746989227317 \mathrm{e}-002$ & $-8.090268621265134 \mathrm{e}-002$ \\
\hline 10 & $-4.416639244876935 \mathrm{e}-002$ & $4.945017933994644 \mathrm{e}-002$ \\
\hline 11 & $2.738672428390126 \mathrm{e}-002$ & $-3.142658732964170 \mathrm{e}-002$ \\
\hline 12 & $-1.832442604348075 \mathrm{e}-002$ & $1.836068887085309 \mathrm{e}-002$ \\
\hline 13 & & $-9.893828004585433 \mathrm{e}-003$ \\
\hline 14 & & $5.587413566494689 \mathrm{e}-003$ \\
\hline
\end{tabular}

$$
H_{1}(1)=G_{1}(1)=0
$$

Substituting (2.7) and (2.8) into (4.1) and (4.2), one gets a set of linear equations that have to be satisfied. The problem is a constrained nonlinear optimization problem with linear constraints, which can be solved using the subroutine NCONF in the IMSL library. If all the freedom is used to maximize the number of zeros at $z=-1$, then (4.1) becomes a system of linear equations. Alternatively, if the least squares error function is used, the objective function becomes quadratic, and the problem is recognized as a quadratic programming problem with equality constraints. Again, this can be solved numerically with relative ease. In this section, we will limit ourselves to a class of wavelet bases with $K_{0}=1$ using the methods introduced in Section III. The advantage is that it is an analytic solution and is very easy to apply. More precisely, we only impose one zero at $z=-1$ for $H_{0}(z)$ and $G_{0}(z)$.

Due to the special structure of (2.7), it can be seen that the regularity condition in (4.1) is satisfied with $K_{0}=1$ when $\left.\beta(z)\right|_{z=1}=1$. If $\beta(z)$ is an even length filter, its odd part $\beta_{o}(z)$, is a type-IV FIR function, which is equal to zero at $z=1$. Therefore, the constraint $\left.\beta(z)\right|_{z=1}=1$ is simplified to $\left.\beta_{e}(z)\right|_{z=1}=1$ for its even part. Since this constraint cannot be incorporated directly into the Parks-McClellan algorithm [24], it is imposed into $\beta_{e}(z)$ by the following factorization:

$$
\beta_{e}(z)=\left(1-z^{-1}\right) \cdot \hat{\beta}_{e}(z)+\frac{1}{2}\left(1+z^{-1}\right) \cdot z^{-S_{\beta}}
$$

where $S_{\beta}=\left(N_{\beta}-2\right) / 2$, and $\hat{\beta}_{e}(z)$ is a type-III linear-phase filter with length $\hat{N}_{\beta}=N_{\beta}-1$. Equation (4.3) is obtained by observing that $\hat{\beta}_{e}(z)$ is symmetric so that its coefficients, except the two around the center of symmetry, can be written as a product of a type-III linear-phase function and $\left(1-z^{-1}\right)$. The remaining two coefficients are multiple of $\left(1+z^{-1}\right) \cdot z^{-S_{\beta}}$. Using a scale factor of $1 / 2,(4.3)$ guarantees that the required condition $\left.\beta_{e}(z)\right|_{z=1}=1$ is satisfied. Since $\hat{\beta}_{e}(z)$ is a type-III linear-phase function, it can be written as

$$
\hat{\beta}_{e}\left(e^{j \omega}\right)=j \cdot e^{-j \omega\left(\hat{N}_{\beta}-1\right) / 2} \sin (\omega) \cdot \hat{P}_{e}^{(\beta)}(\cos (\omega))
$$

where

$$
\hat{P}_{e}^{(\beta)}(\cos (\omega))=\sum_{k=0}^{\hat{L}_{\beta}} a_{k}(\cos (\omega))^{k}, \quad \hat{L}_{\beta}=\left(\hat{N}_{\beta}-3\right) / 2 .
$$

Substituting (4.3) and (4.4) into (2.7), one obtains the error function as follows:

$$
E_{0}(\omega)=\frac{1}{2} e^{-j 2 \omega N}\left\{e^{-j 2 \omega N_{\Delta}} P^{(\beta)}\left(e^{j \omega}\right)-1\right\}
$$




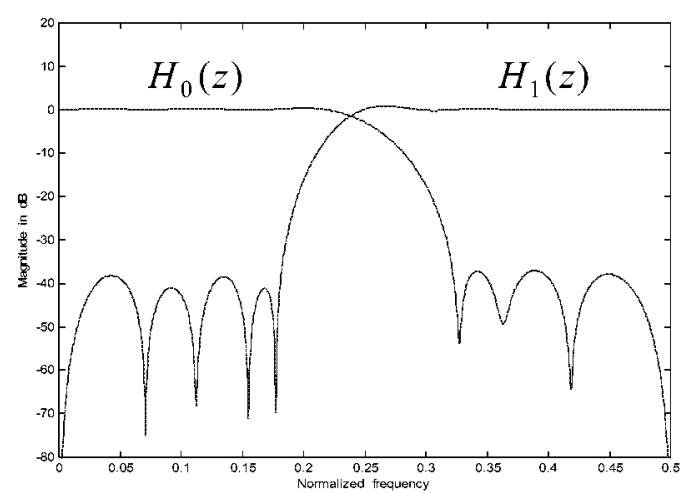

(a)

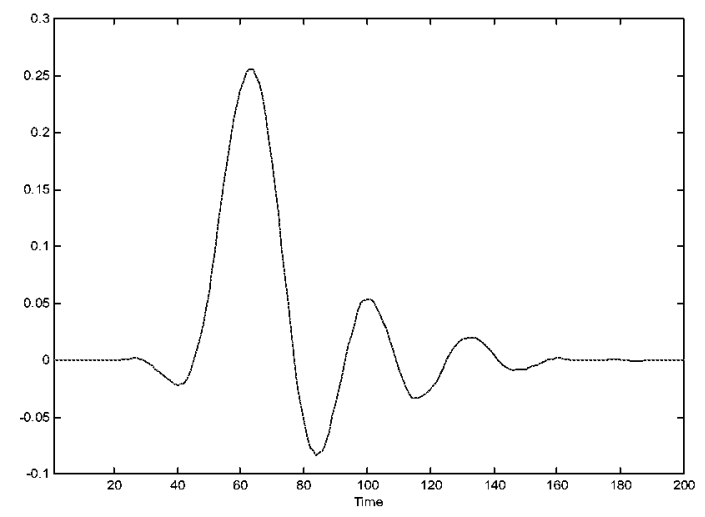

(c)

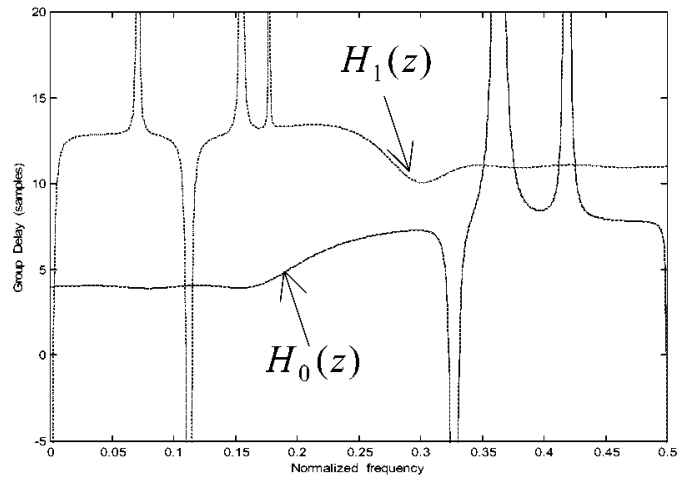

(b)

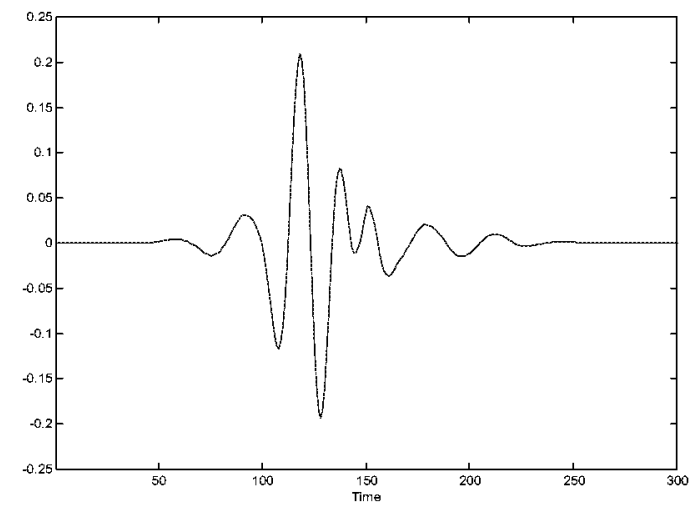

(d)

Fig. 6. Analysis filters with wavelet bases (example 3.3) designed by the Remez exchange algorithm (a) magnitude responses of analysis filters $H_{0}(z)$ and $H_{1}(z)$; (b) their group delay responses. (c) analysis scaling function; (d) analysis wavelet function.

where

$$
\begin{aligned}
P^{(\beta)}\left(e^{j \omega}\right)= & \left\{\cos (\omega)-2 \sin (\omega) \cdot \sin (2 \omega) \hat{P}_{e}^{(\beta)}(\cos (2 \omega))\right\} \\
& +j \cdot \sin \omega \cdot P_{o}^{(\beta)}(\cos (2 \omega))
\end{aligned}
$$

$N_{\Delta}=\left(N_{\beta} / 2\right)-N$, and $P_{o}^{(\beta)}(\cos (\omega))$ are given by (3.7b). If the Chebyshev or minimax problem is solved as two independent real Chebyshev approximations, the desired functions $\hat{P}_{e}^{(\beta)}(\cos (2 \omega))$ and $P_{o}^{(\beta)}(\cos (2 \omega))$ are given by

and

$$
\tilde{P}_{e}^{(\beta)}(\cos (2 \omega))=\frac{\cos (\omega)-\cos \left(2 N_{\Delta} \omega\right)}{2 \sin (\omega) \cdot \sin (2 \omega)}
$$

$$
\tilde{P}_{o}^{(\beta)}(\cos (2 \omega))=\frac{\sin \left(2 \omega N_{\Delta}\right)}{\sin (\omega)}, \quad 0<\omega<\pi / 2
$$

This can be solved using the Remez exchange algorithm with weighting functions

$$
\begin{aligned}
& W_{e}(x)=2 \sin (0.5 \cdot \arccos (x)) \cdot \sin (\arccos (x)) \\
& W_{o}(x)=\sin (0.5 \cdot \arccos (x))(x=\cos (2 \omega)) .
\end{aligned}
$$

We now consider the design of the highpass filter. It can be seen from (2.7) that $\left.H_{0}(z)\right|_{z=1}=1$ when the condition $\left.\beta(z)\right|_{z=1}=1$ is incorporated. Therefore, the highpass filter $H_{1}(z)$ will be zero at $z=1$ when $\left.\alpha(z)\right|_{z=1}=1$. Again, using the property $\left.\alpha_{o}(z)\right|_{z=1}=0$ and the following factorization for $\alpha_{e}(z)$

$$
\alpha_{e}(z)=\left(1-z^{-1}\right) \cdot \hat{\alpha}_{e}(z)+\frac{1}{2}\left(1+z^{-1}\right) \cdot z^{-S_{\alpha}}
$$

where

$$
\begin{gathered}
\hat{\alpha}_{e}\left(e^{j \omega}\right)=j \cdot e^{-j \omega\left(\hat{N}_{\alpha}-1\right) / 2} \sin (\omega) \cdot \hat{P}_{e}^{(\alpha)}(\cos (\omega)) \\
\hat{N}_{\alpha}=N_{\alpha}-1 \\
\hat{P}_{e}^{(\alpha)}(\cos (\omega))=\sum_{k=0}^{\hat{L}_{\alpha}} \tilde{a}_{k}(\cos (\omega))^{k} \\
S_{\alpha}=\left(N_{\alpha}-2\right) / 2, \quad \hat{L}_{\alpha}=\left(\hat{N}_{\alpha}-3\right) / 2
\end{gathered}
$$

the required regularity condition can be incorporated. Let $P^{(\alpha)}\left(e^{j \omega}\right)$ be given by

$$
\begin{aligned}
P^{(\alpha)}\left(e^{j \omega}\right)=\{ & \cos (\omega)-2 \sin (\omega) \cdot \sin (2 \omega) \cdot \hat{P}_{e}^{(\alpha)}(\cos (2 \omega)) \\
& \left.+j \cdot \sin (\omega) \cdot P_{o}^{(\alpha)}(\cos (2 \omega))\right\} .
\end{aligned}
$$


TABLE IV

SOPOT COEFFICIENTS FOR EXAMPLES 5.1 AND 5.2.

\begin{tabular}{c|c|c|c|c}
\hline & \multicolumn{2}{|c|}{ Example 5.1 } & \multicolumn{2}{c}{ Example 5.2} \\
\hline $\mathrm{n}$ & $\beta(z)$ & $\alpha(z)$ & $\beta(z)$ & $\alpha(z)$ \\
\hline 0 & $-2^{-4}-2^{-6}-2^{-8}$ & $-2^{-6}+2^{-9}$ & $2^{-5}+2^{-7}+2^{-8}$ & $-2^{-8}+2^{-10}$ \\
\hline 1 & $2^{-1}+2^{-7}+2^{-8}$ & $2^{-4}$ & $-2^{-3}+2^{-8}$ & $2^{-6}+2^{-8}+2^{-10}$ \\
\hline 2 & $2^{-1}+2^{-2}-2^{-8}$ & $-2^{-3}-2^{-6}-2^{-7}$ & $2^{-1}+2^{-4}-2^{-6}$ & $-2^{-5}-2^{-6}$ \\
\hline 3 & $-2^{-2}-2^{-5}$ & $2^{-1}+2^{-4}+2^{-5}$ & $2^{-1}+2^{-2}-2^{-5}$ & $2^{-4}+2^{-6}+2^{-9}$ \\
\hline 4 & $2^{-3}+2^{-5}$ & $2^{-1}+2^{-3}+2^{-5}$ & $-2^{-2}-2^{-5}-2^{-7}$ & $-2^{-2}+2^{-4}+2^{-6}$ \\
\hline 5 & $-2^{-4}-2^{-6}$ & $-2^{-2}+2^{-5}+2^{-6}$ & $2^{-2}-2^{-4}-2^{-8}$ & $2^{-1}+2^{-3}-2^{-6}$ \\
\hline 6 & $2^{-5}$ & $2^{-4}+2^{-5}$ & $-2^{-3}-2^{-7}$ & $2^{-1}+2^{-3}+2^{-5}$ \\
\hline 7 & $-2^{-7}$ & $-2^{-5}-2^{-6}$ & $2^{-3}-2^{-5}$ & $-2^{-2}+2^{-5}+2^{-8}$ \\
\hline 8 & & $2^{-6}+2^{-9}$ & $-2^{-4}-2^{-10}$ & $2^{-3}-2^{-8}$ \\
\hline 9 & & $-2^{-8}$ & $2^{-5}+2^{-7}$ & $-2^{-4}-2^{-7}-2^{-8}$ \\
\hline 10 & & & $-2^{-6}-2^{-7}$ & $2^{-4}-2^{-6}-2^{-9}$ \\
\hline 11 & & & $2^{-7}+2^{-8}$ & $-2^{-5}+2^{-8}$ \\
\hline 12 & & & & $2^{-6}-2^{-9}$ \\
\hline 13 & & & & \\
\hline
\end{tabular}

Following the same approach in designing the lowpass filter, the desired responses of $\hat{P}_{e}^{(\alpha)}(\cos \omega)$ and $P_{o}^{(\alpha)}(\cos \omega)$ are shown to be

$$
\begin{aligned}
\tilde{P}_{e}^{(\alpha)}(\cos (2 \omega))= & \frac{1}{2 \sin (\omega) \sin (2 \omega)} \\
& \cdot\left[\cos (\omega)-\frac{\operatorname{Re}\left(A^{*}\left(e^{j \omega}\right) e^{j 2 \omega M_{\Delta}}\right)}{\left|A\left(e^{j \omega}\right)\right|^{2}}\right] \\
\tilde{P}_{o}^{(\alpha)}(\cos (2 \omega))= & \frac{\operatorname{Im}\left(A^{*}\left(e^{j \omega}\right) e^{j 2 \omega M_{\Delta}}\right)}{\left|A\left(e^{j \omega}\right)\right|^{2} \sin (\omega)}, \quad 0<\omega<\pi / 2
\end{aligned}
$$

where $A\left(e^{j \omega}\right)$ is the complex envelope of $H_{0}\left(e^{j \omega}\right)$ given by (3.22). Again, the optimal minimax solution is similar to that in (4.9) with weighting function given by

and

$$
\begin{gathered}
W_{e}(x)=2 \sin (0.5 \cdot \arccos (x)) \cdot \sin (\arccos (x)) \\
\cdot\left|A\left(e^{j 0.5 \arccos (x)}\right)\right|^{2}
\end{gathered}
$$

$$
\begin{aligned}
W_{o}(x)= & \sin (0.5 \cdot \arccos (x)) \cdot\left|A\left(e^{j 0.5 \arccos (x)}\right)\right|^{2} \\
& (x=\cos (2 \omega)) .
\end{aligned}
$$

This design algorithm can also be generalized to the case where the length of $\beta(z)$ is odd. The detail is given in Appendix B. Due to page limitations, derivation of the least squares design is omitted here. It should be noted that the proposed method is also applicable to the design of wavelet bases with linear phase.

Example 4.1: In this example, we will design a wavelet filterbank with the same parameters as Example 3.1 using the proposed method. In particular, the lengths of the FIR functions $\beta(z)$ and $\alpha(z)$ are $N_{\beta}=8$ and $N_{\alpha}=10$, respectively. The low-delay parameters $N_{\Delta}$ and $M_{\Delta}$ are also selected as 2 and 1 . The system delay is maintained as 15 samples. Fig. 6(a) and (b) plot the magnitude and group delay responses of $H_{0}(z)$ (solid line) and $H_{1}(z)$ (dashed line). It is observed that the frequency performance of $H_{0}(z)$ and $H_{1}(z)$ of the wavelet filterbank is comparable with that in Example 3.1, except those zeros have been imposed at $\omega=\pi$ for $H_{0}(z)$ and $\omega=0$ for $H_{1}(z)$. The analysis scaling and wavelet functions of these low-delay wavelet filterbanks are shown in Fig. 6(c) and (d), which are very smooth. The coefficients of $\beta(z)$ and $\alpha(z)$ are also listed in Table I.

\section{MUltiPlierless LOW-DELAY FIR FILTER BANKS}

In this section, we will study the multiplierless implementation of the proposed low-delay FIR filterbanks using SOPOT coefficients and the GA. The design method proposed in the previous sections is very efficient for designing low-delay FIR filterbanks with real-valued coefficients. Since the filterbank is structurally PR, the simplest way to obtain a PR filterbank with SOPOT coefficients is to round the coefficients obtained in Sections III and IV to the nearest SOPOT coefficients. It is, however, a suboptimal solution. A number of methods have been proposed for designing FIR filters with SOPOT coefficients. A classical work is the integer programming method proposed by 


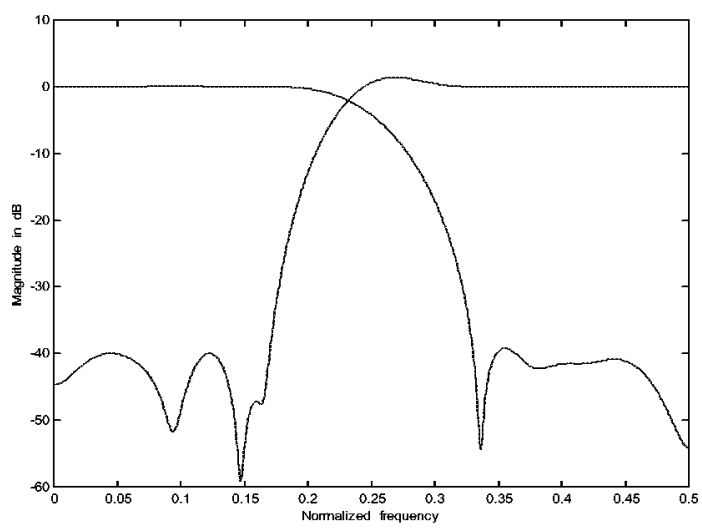

Fig. 7. Analysis filters of Example 5.1 designed by GA. Magnitude responses of analysis filters $H_{0}(z)$ and $H_{1}(z)$.

Lim [33]. Other heuristic methods such as simulated annealing [34] and GA [35] have also been proposed as alternatives to the problem. These heuristic techniques are, in general, very easy to use and are able to a yield reasonably good solution even when the objective function is nonsmooth with complicated (inequalities) constraints. An important drawback, however, is the long computational time of the algorithms, which depend on a number of issues such as the strategy used, coding style, and the initial population, etc. In [35], the GA is used to obtain the SOPOT coefficients for the lattice coefficients in a multiplierless two-channel orthogonal filterbank derived from the lossless lattice structure [2]. In this paper, the GA is also employed to search for the SOPOT coefficients of the functions $\beta(z)$ and $\alpha(z)$. More precisely, the coefficients of $\beta(z)$ and $\alpha(z)$ are represented as

$$
\begin{aligned}
h(n)=\sum_{k=1}^{p_{n}} a_{k} \cdot 2^{b_{k}}, \quad a_{k} \in\{-1,1\} \\
b_{k} \in\{-\ell, \cdots,-1,0,1, \cdots, \ell\}
\end{aligned}
$$

where $\ell$ is a positive integer, and its value determines the range of the coefficients. $p_{n}$ is the number of terms of the $n$th coefficient. Normally, $p_{n}$ is limited to a small number, and the multiplication of such SOPOT coefficients can be implemented with simple shifts and additions. The objective function we minimize is

$$
E_{k}\left(H_{k}\right)=\max \left\|H_{k}(\omega)-\tilde{H}_{k}(\omega)\right\|, \quad k=0,1, \quad \omega \in[0, \pi]
$$

where $\tilde{H}_{k}(\omega)$ is the desired frequency response. Various aspects on the implementation of GA can be found in [37] and the references therein.

Example 5.1: In this example, a multiplierless low-delay filterbank with the same specification as Example 3.1 is designed using the GA. The cut-off frequencies are $\omega_{p}=0.34 \pi$ and $\omega_{s}=0.66 \pi$. The SOPOT coefficients of the filterbank obtained are shown in Table IV. Fig. 7 shows the frequency responses of the multiplierless PR filterbank. The stopband attenuation of

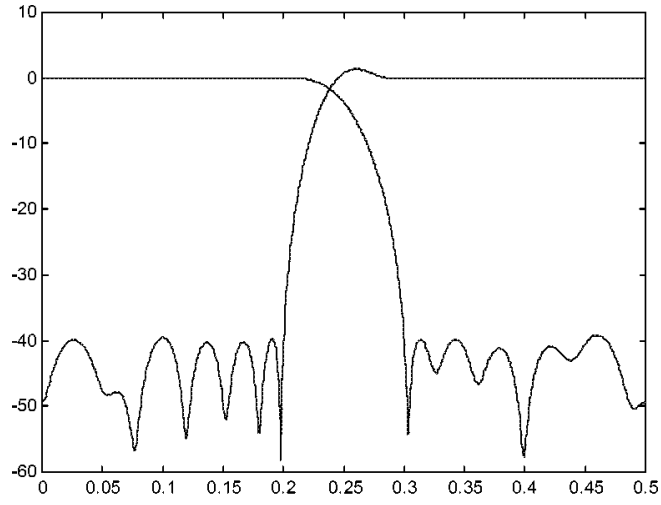

Fig. 8. Analysis filters of Example 5.2 designed by GA: magnitude responses of analysis filters $H_{0}(z)$ and $H_{1}(z)$.

$H_{0}(z)$ and $H_{1}(z)$ are, respectively, $39.2 \mathrm{~dB}$ and $40 \mathrm{~dB}$, which are very close to their real-valued counterparts. The averaged number of terms used per coefficient is only 2.2. The total arithmetic complexities for implementing $\beta(z)$ and $\alpha(z)$ are 37 additions and 39 shifts. Counting the two more additions in the filterbank, the total arithmetic complexity is 39 additions and 39 shifts in the decimated domain or 19.5 additions and 19.5 shifts in the original sampling rate. The number of shift operations can further be reduced if additional memory locations are used to store the shifted immediate data. These results demonstrate the low implementation complexity and good performance of the proposed multiplierless filterbank. It is also found that the GA, although requiring more computational time, is very effective in finding the SOPOT coefficients of the multiplierless low-delay filterbanks with good frequency characteristics.

Example 5.2: In this example, another multiplierless lowdelay filterbank is designed using the GA. The specifications are $\omega_{p}=0.4 \pi$ and $\omega_{s}=0.6 \pi . N=3, M=8, N_{\beta}=12$, and $N_{\alpha}=14$. The SOPOT coefficients obtained are also shown in Table IV. Fig. 8 shows the frequency responses of the multiplierless filterbank. Both the stopband attenuation of $H_{0}(z)$ and $H_{1}(z)$ are $39 \mathrm{~dB}$, which are very close to their real-valued counterparts. The average number of terms per coefficient is 2.5 . The total arithmetic complexity required in the original sampling rate is 32 additions and 32 shifts. The number of shifts can be reduced if additional memory locations are used to store the shifted immediate data. These results again demonstrate the effectiveness of the GA and the low implementation complexity of the multiplierless low-delay filterbank. More design examples can be found in [36]. To obtain the results in Examples 5.1 and 5.2 by GA, it requires approximately 20 to $40 \mathrm{~min}$, respectively, on a Pentium II $350 \mathrm{MHz}$ personal computer if no initial guesses for $\alpha(z)$ and $\beta(z)$ are used. The computational time can, however, be greatly reduced if $\alpha(z)$ and $\beta(z)$ in Examples 3.1 and 4.1 are quantized to SOPOT coefficients and included in the initial population.

\section{CONCLUSION}

In this paper, a new approach for designing two-channel PR FIR filterbanks with low system delay is presented. It is based 
on the use of nonlinear-phase FIR functions in the PR structure previously proposed by Phoong et al. [9]. Because the linearphase requirement is relaxed, the lengths of $\beta(z)$ and $\alpha(z)$ are no longer restricted by the delay parameters of the filterbanks. Hence, higher stopband attenuation can be achieved at low system delay. The design of the proposed low-delay filterbanks is formulated as a complex polynomial approximation problem, which is solved by the Remez exchange algorithm or analytic formula with very low complexity. The generalization of the present approach to the design of a class of wavelet bases associated with this low-delay filterbank and its multiplier-less implementation using the sum of powers-of-two coefficients are also studied.

APPENDIX A

DESIGN OF $\beta(z)$ AND $\alpha(z)$ WITH ODD LENGTH

When the length of $\beta(z)$ is odd, it can be written as a sum of type-I and type-III FIR functions as follows:

$$
\begin{aligned}
& \beta_{e}\left(e^{j \omega}\right)=e^{-j \omega\left(N_{\beta}-1\right) / 2} \cdot P_{e}^{(\beta)}(\cos (\omega)) \\
& \beta_{o}\left(e^{j \omega}\right)=j e^{-j \omega\left(N_{\beta}-1\right) / 2} \sin (\omega) \cdot P_{o}^{(\beta)}(\cos (\omega))
\end{aligned}
$$

where

$$
\begin{aligned}
& P_{e}^{(\beta)}(\cos (\omega))=\sum_{k=0}^{L_{\beta_{e}}} a_{k}(\cos (\omega))^{k}, \quad L_{\beta_{e}}=\left(N_{\beta}-1\right) / 2 \\
& P_{o}^{(\beta)}(\cos (\omega))=\sum_{k=0}^{L_{\beta_{o}}} b_{k}(\cos (\omega))^{k}, \quad L_{\beta_{o}}=\left(N_{\beta}-3\right) / 2 \text {. }
\end{aligned}
$$

The desired magnitude response of $P^{(\beta)}\left(e^{j \omega}\right)=$ $P_{e}^{(\beta)}(\cos (2 \omega))+j P_{o}^{(\beta)}(\cos (2 \omega))$ is still given by (3.9). If the complex Chebyshev approximation is solved using two real approximations as in Section III-A, the ideal responses in (3.10) are modified to

$$
\begin{aligned}
& \hat{P}_{e}^{(\beta)}(\cos (2 \omega))=\cos \left(2 \omega N_{\Delta}\right), \\
& \hat{P}_{o}^{(\beta)}(\cos (2 \omega))=\frac{\sin \left(2 \omega N_{\Delta}\right)}{\sin (2 \omega)}, \quad 0<\omega<\pi / 2
\end{aligned}
$$

with weighting functions

$W_{e}(x)=1$, and $W_{o}(x)=\sin (\arccos (x))(x=\cos (2 \omega))$

A factorization similar to (A1.1) also exists when the length of $\alpha(z)$ is odd. In fact, the desired response of $P^{(\alpha)}\left(e^{j \omega}\right)=$ $P_{e}^{(\alpha)}(\cos (2 \omega))+j P_{o}^{(\alpha)}(\cos (2 \omega))$ is still given by (3.24), where

$$
\begin{array}{ll}
P_{e}^{(\alpha)}(\cos (\omega))=\sum_{k=0}^{L_{\alpha_{e}}} \tilde{a}_{k}(\cos (\omega))^{k}, & L_{\alpha_{e}}=\left(N_{\alpha}-1\right) / 2 \\
P_{o}^{(\alpha)}(\cos (\omega))=\sum_{k=0}^{L_{\alpha_{o}}} \tilde{b}_{k}(\cos (\omega))^{k}, & L_{\alpha_{o}}=\left(N_{\alpha}-3\right) / 2
\end{array}
$$

The ideal responses of $P_{e}^{(\alpha)}(\cos (2 \omega))$ and $P_{o}^{(\alpha)}(\cos (2 \omega))$, using separate real and imaginary parts approximation, are

$$
\begin{aligned}
\hat{P}_{e}^{(\alpha)}(\cos (2 \omega)) & =\operatorname{Re}\left(A^{-1}\left(e^{j \omega}\right) e^{j 2 \omega M_{\Delta}}\right) \\
& =\frac{\operatorname{Re}\left(A^{*}\left(e^{j \omega}\right) e^{j 2 \omega M_{\Delta}}\right)}{\left|A\left(e^{j \omega}\right)\right|^{2}} \\
\hat{P}_{o}^{(\alpha)}(\cos (2 \omega)) & =\frac{\operatorname{Im}\left(A^{-1}\left(e^{j \omega}\right) e^{j 2 M_{\Delta} \omega}\right)}{\sin (2 \omega)} \\
& =\frac{\operatorname{Im}\left(A^{*}\left(e^{j \omega}\right) e^{j 2 M_{\Delta}}\right)}{\left|A\left(e^{j \omega}\right)\right|^{2} \sin (2 \omega)}, \quad 0<\omega<\pi / 2 .
\end{aligned}
$$

The corresponding weighting functions in the Remez exchange algorithm are

$$
\begin{aligned}
& \tilde{W}_{e}(x)=\left|A\left(e^{j 0.5 \arccos (x)}\right)\right|^{2} \\
& \text { and } \\
& \tilde{W}_{o}(x)=\sin (\arccos (x))\left|A\left(e^{j 0.5 \arccos (x)}\right)\right|^{2}(x=\cos (2 \omega)) \cdot
\end{aligned}
$$

It should be noted that because $N_{\beta}$ and $N_{\alpha}$ are odd integers, the low delay parameters $N_{\Delta}$ and $M_{\Delta}$ are half integers. Due to page limitations, detail derivation of the least squares solution is omitted. It can be derived similar to the even length case described in Sections III-A and III-B.

\section{APPENDIX B}

\section{WAVELET BASES FOR $\beta(z)$ AND $\alpha(z)$ WITH ODD LENGTH}

When the length of $\beta(z)$ is odd, it can be written as a sum of a type-I FIR function $\beta_{e}(z)$ and a type-III FIR function $\beta_{o}(z)$. Since a type-III FIR function has a zero at $z=0, \beta_{e}(z)$ can be factored using the same method in (4.3)

$$
\beta_{e}(z)=\left(1-z^{-1}\right) \cdot \hat{\beta}_{e}(z)+z^{-S_{\beta}}
$$

where $S_{\beta}=\left(N_{\beta}-1\right) / 2$, and $\hat{\beta}_{e}(z)$ is a type-IV FIR function with length $\hat{N}_{\beta}=N_{\beta}-1$. If the Chebyshev problem is solved by using two independent real Chebyshev approximations, the desired magnitude responses of $\beta_{e}(z)$ and $\beta_{o}(z)$ in (4.7) and (4.8) are modified to

$$
\begin{aligned}
& \tilde{P}_{e}^{(\beta)}(\cos (2 \omega))=\frac{1-\cos \left(2 N_{\Delta} \omega\right)}{2 \sin ^{2}(\omega)} \\
& \tilde{P}_{o}^{(\beta)}(\cos (2 \omega))=\frac{\sin \left(2 \omega N_{\Delta}\right)}{\sin (2 \omega)}, \quad 0<\omega<\pi / 2
\end{aligned}
$$

with weighting functions

$$
W_{e}(x)=2 \sin ^{2}(0.5 \arccos (x))
$$

and

$$
W_{o}(x)=\sin (\arccos (x))(x=\cos (2 \omega)) .
$$


Similar modifications can be made when the length of $\alpha(z)$ is odd. The desired magnitude responses of $\alpha(z)$ in (4.11) and (4.12) are modified to

$$
\begin{aligned}
& \tilde{P}_{e}^{(\alpha)}(\cos (2 \omega))=\frac{1}{2 \sin ^{2} \omega}\left[1-\frac{\operatorname{Re}\left(A^{*}\left(e^{j \omega}\right) e^{j 2 \omega M_{\Delta}}\right)}{\left|A\left(e^{j \omega}\right)\right|^{2}}\right] \\
& \tilde{P}_{o}^{(\alpha)}(\cos (2 \omega))=\frac{\operatorname{Im}\left(A^{*}\left(e^{j \omega}\right) e^{j 2 \omega M_{\Delta}}\right)}{\left|A\left(e^{j \omega}\right)\right|^{2} \sin (2 \omega)}, \quad 0<\omega<\pi / 2
\end{aligned}
$$

with weighting functions

$$
W_{e}(x)=2 \sin ^{2}(0.5 \arccos (x)) \cdot\left|A\left(e^{j 0.5 \arccos (x)}\right)\right|^{2}
$$

and

$$
W_{o}(x)=\sin (\arccos (x)) \cdot\left|A\left(e^{j 0.5 \arccos (x)}\right)\right|^{2}(x=\cos (2 \omega)) \text {. }
$$

\section{REFERENCES}

[1] M. J. T. Smith and T. P. Barnwell III, "Exact reconstruction techniques for tree structured subband coders," IEEE Trans. Acoust., Speech, Signal Processing, vol. ASSP-34, pp. 434-441, June 1986.

[2] P. P. Vaidyanathan, Multirate Systems and filterbanks. Englewood Cliffs, NJ: Prentice-Hall, 1992.

[3] — - "Multirate digital filters, filterbanks, polyphase networks and applications: A tutorial," Proc. IEEE, vol. 78, pp. 56-93, Jan. 1990.

[4] A. N. Akansu and R. A. Haddad, Multiresolution Signal Decomposition: Transforms, Subbands, and Wavelets. Boston, MA: Academic, 1992.

[5] M. Vetterli, "A theory of multirate filterbanks," IEEE Trans. Acoust., Speech, Signal Processing, vol. ASSP-35, pp. 356-372, Mar. 1987.

[6] C. W. Kim and R. Ansari, "FIR/IIR exact reconstruction filterbank with applications to subband coding of images," in Proc. 34th Midwest CAS Symp., vol. 1, May 1991, pp. 227-230.

[7] H. Kiya, M. Yae, and M. Iwahashi, "A linear phase two- channel filterbank allowing perfect reconstruction," Proc. IEEE ISCAS, vol. 2, pp. 951-954, May 1992.

[8] T. Q. Nguyen and P. P. Vaidyanathan, "Two-channel perfect-reconstruction FIR QMF structures which yield linear-phase analysis and synthesis filters," IEEE Trans. Acoust., Speech, Signal Processing, vol. 37, pp. 676-690, May 1989.

[9] S. M. Phoong, C. W. Kim, P. P. Vaidyanathan, and R. Ansari, "A new class of two-channel biothogonal filterbanks and wavelet bases," IEEE Trans. Signal Processing, vol. 43, pp. 649-664, Mar. 1995.

[10] K. Nayebi, T. P. Barnwell III, and M. J. T. Smith, "Low delay FIR filterbanks: Design and evaluation," IEEE Trans. Signal Processing, vol. 42, pp. 24-31, Jan. 1994.

[11] E. Abdel-Raheem, F. El-Guibaly, and A. Antoniou, "Design of lowdelay FIR QMF banks using the Lagrange-multiplier approach," Electron. Lett., vol. 30, no. 12, pp. 924-926, June 1994.

[12] R. Gandhi and S. K. Mitra, "Design of two-channel low delay perfect reconstruction filterbanks," in Proc. 32nd Int. Asilomar Conf. Signals, Syst., Comput., vol. 2, Nov. 1998, pp. 1655-1659.

[13] E. Abdel-Raheem, F. El-Guibaly, and A. Antoniou, "Design of lowdelay perfect-reconstruction FIR filterbanks for tree-structured subband coders," in Proc. IEEE ISCAS, vol. 2, May 1995, pp. 969-972.

[14] G. Schuller and M. J. T. Smith, "A new algorithm for efficient low delay filterbank design," in Proc. IEEE ICASSP, vol. 2, May 1995, pp. $1472-1475$.

[15] A. Al-Adnani, R. Chapman, and T. S. Durrani, "Time-domain design of low delay wavelet transforms," Electron. Lett., vol. 29, no. 25, pp. 2177-2178, Dec. 1993.

[16] H. Ochi, M. Ohta, and S. Kinjo, "Linear programming design of twochannel perfect-reconstruction biorthogonal filterbanks-Linear-phase and low-delay," in Proc. IEEE 40th Midwest Symp. Circuits Syst., vol. 2, Aug. 1997, pp. 969-972.

[17] G. Schuller and M. J. T. Smith, "Efficient low delay filterbanks," in Proc. IEEE 6th DSP Workshop, 1994, pp. 231-234.

[18] H. Xu, W. S. Lu, and A. Antoniou, "A new approach for the design of low-delay quadrature mirror filterbanks," in Proc. 28th Int. Asilomar Conf. Signals, Syst., Comput., vol. 2, Nov. 1994, pp. 1001-1004.
[19] — - "An improved method for the design of FIR quadrature mirror-image filterbanks," IEEE Trans. Signal Processing, vol. 46, pp. 1275-1281, May 1998.

[20] S. J. Yang, J. H. Lee, and B. C. Chieu, "Minimax design of two-channel low-delay perfect-reconstruction FIR filterbanks," Proc. ,Isnt. Elect. Eng., Vis. Image Signal Process., vol. 146, no. 1, pp. 15-24, Feb. 1999.

[21] C. S. Burrus and J. A. Barreto, "Least p-power error design of FIR filters," in Proc. IEEE ISCAS, vol. 2, 1992, pp. 545-548.

[22] A. S. Alkhairy, K. G. Christian, and J. S. Lim, "Design and characterization of optimal FIR filters with arbitrary phase," IEEE Trans. Signal Processing, vol. 41, pp. 559-572, Feb. 1993.

[23] M. Lang and J. Bamberger, "Nonlinear phase FIR filter design with minimum LS error and additional constraints," in Proc. IEEE ICASSP, vol. 3, Apr. 1993, pp. 57-60.

[24] L. J. Karam and J. H. McClellan, "Complex Chebyshev approximation for FIR filter design," IEEE Trans. Circuits Syst. II, vol. 42, pp. 207-216, Mar. 1995.

[25] S. C. Pei and J. J. Shyu, "Design of complex FIR filters with arbitrary complex frequency responses by two real Chebyshev approximations," IEEE Trans. Circuits Syst. I, vol. 44, pp. 170-174, Feb. 1997.

[26] M. Z. Komodromos, S. F. Russell, and P. T. P. Tang, "Design of FIR filters with complex desired frequency response using a generalized Remez algorithm," IEEE Trans. Circuits Syst. II, vol. 42, pp. 274-278, Apr. 1995.

[27] W. Lertniphonphun and J. H. McClellan, "Complex frequency response FIR filter design," in Proc. IEEE ICASSP, vol. 3, May 1998, pp. 1301-1304.

[28] A. Cohen, I. Daubechies, and J. Feauveau, "Biorthogonal bases of compactly supported wavelets," Commun. Pure Appl. Math., vol. XLV, pp. 485-560, 1992.

[29] M. Vetterli and C. Herley, "Wavelets and filterbanks: Theory and design," IEEE Trans. Signal Processing, vol. 40, pp. 2207-2232, Sept. 1992.

[30] M. Antonini, M. Barlaud, P. Mathieu, and I. Daubechies, "Imaging coding using wavelet transform," IEEE Trans. Image Processing, vol. 1, pp. 255-220, Apr. 1992.

[31] S. C. Chan, J. S. Mao, and K. L. Ho, "A new design method for twochannel perfect reconstruction IIR filterbanks," J. Opt. Eng., to be published.

[32] P. A. Naylor, O. Tanrikulu, and A. G. Constantinides, "Subband adaptive filtering for acoustic echo control using allpass polyphase IIR filterbanks," IEEE Trans. Speech Audio Processing, vol. 6, pp. 143-155, Mar. 1998.

[33] Y. C. Lim and S. R. Parker, "FIR filter design over a discrete power-of-two coefficient space," IEEE Trans. Acoust., Speech, Signal Processing, vol. ASSP-31, pp. 583-591, June 1983.

[34] N. Benvenuto, M. Marchesi, and A. Uncini, "Applications of simulated annealing for the design of special digital filters," IEEE Trans. Signal Processing, vol. 40, pp. 323-332, Feb. 1992.

[35] S. Sriranganathan, D. R. Bull, and D. W. Redmill, "The design of low complexity two-channel lattice-structure perfect-reconstruction filterbanks using genetic algorithms," in Proc. IEEE ISCAS, vol. 4, 1997, pp. 2393-2396

[36] W. Liu and S. C. Chan, "Design of multiplier-less two-channel perfect reconstruction filterbanks using genetic algorithm," Dept. Elect. Electron. Eng., The Univ. Hong Kong, Intern. Rep., Oct. 1999.

[37] K. S. Tang, K. F. Man, S. Kwong, and Q. He, "Genetic algorithms and their applications," IEEE Signal Processing Mag., pp. 22-37, Nov. 1996.

[38] A. V. Oppenheim, R. W. Schafer, and J. R. Buck, Discrete-Time Signal Processing. Upper Saddle River, NJ: Prentice-Hall, 1999.

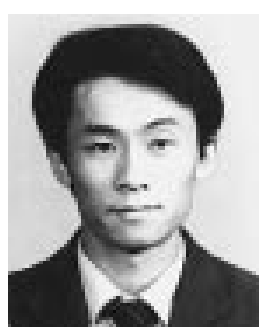

J. S. Mao received the B.S. degree in electronic engineering from Shanghai Jiao Tong University, Shanghai, China, in 1989, the M.Eng. degree from the University of Electronic Science and Technology of China, Chengdu, in 1992, and the Ph.D. degree from the University of Hong Kong in 2000.

From April 1992 to October 1995, he was a Lecturer at the University of Electronic Science and Technology of China. He served as a Research Assistant with the University of Hong Kong from November 1995 to April 2000. He is now a Research Fellow at the University of Victoria, Victoria, BC, Canada. His main research interests include digital signal processing, multirate filterbank systems, and speech coding. 


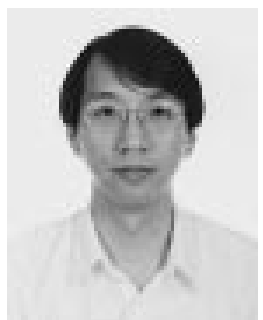

S. C. Chan received the B.Sc. and Ph.D. degrees in electrical engineering from the University of Hong Kong in 1986 and 1992, respectively.

From 1990 to 1994, he was a Lecturer with the Department of Electronic Engineering, City University of Hong Kong. He joined the Department of Electrical and Electronic Engineering, University of Hong Kong, in 1994, where he is now an Associate Professor. His main research interests are in digital signal processing and their applications to communications and data compression. He held visiting positions in Microsoft Research, Redmond, WA, and Microsoft Research, Beijing, China, in 1998 and 1999, respectively.

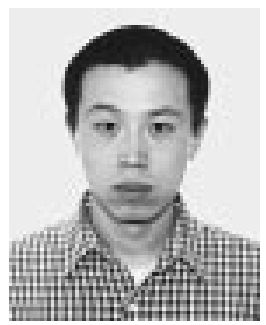

W. Liu was born in Hebei Province, China, on January 22, 1974. He received the B.Sc. degree in space physics, with the honor of "Excellent Graduate," from Peking University, Beijing, China, in 1996. He is currently pursuing the M.Phil. degree at the Department of Electrical and Electronic Engineering, the University of Hong Kong.

His main research interests are in multirate filterbanks and wavelets and their multiplierless design and implementation.

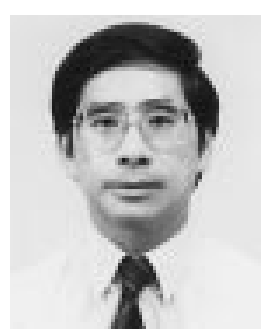

the IEE and the HKIE.
K. L. Ho (M'80) received the B.Sc.(Eng.) and the M.Phil. degrees in electrical engineering from the University of Hong Kong in 1971 and 1973, respectively, and the Ph.D. degree from the University of London, London, U.K., in 1977.

In 1984, he joined the Department of Electrical and Electronic Engineering at the University of Hong Kong. His current research interests include signal processing and communications systems.

Dr. Ho is a chartered engineer of the Engineering Council of the United Kingdom and is a member of 